\title{
Purely leptonic decays of the ground charged vector mesons
}

\author{
Yueling Yang ${ }^{1, \mathrm{a}}{ }_{\mathbb{D}}$, Zhenglin $\mathrm{Li}^{1}$, Kang $\mathrm{Li}^{1}$, Jinshu Huang ${ }^{2}$, Junfeng Sun ${ }^{1, \mathrm{~b}} \mathbb{C}_{\mathbb{C}}$ \\ ${ }^{1}$ Institute of Particle and Nuclear Physics, Henan Normal University, Xinxiang 453007, China \\ 2 School of Physics and Electronic Engineering, Nanyang Normal University, Nanyang 473061, China
}

Received: 2 November 2021 / Accepted: 5 December 2021 / Published online: 16 December 2021

(C) The Author(s) 2021

\begin{abstract}
The study of the purely leptonic decays of the ground charged vector mesons is very interesting and significant in determining the CKM matrix elements, obtaining the decay constant of vector mesons, examining the lepton flavor universality, and searching for new physics beyond the standard model. These purely leptonic decays of the ground charged vector mesons are induced by the weak interactions within the standard model, and usually have very small branching ratios, $\mathcal{B}\left(\rho^{-} \rightarrow \ell^{-} v_{\ell}\right) \sim \mathcal{O}\left(10^{-13}\right)$, $\mathcal{B}\left(K^{*-} \rightarrow \ell^{-} v_{\ell}\right) \sim \mathcal{O}\left(10^{-13}\right), \mathcal{B}\left(D_{d}^{*-} \rightarrow \ell^{-} v_{\ell}\right) \sim \mathcal{O}\left(10^{-10}\right)$, $\mathcal{B}\left(B_{u}^{*-} \rightarrow \ell^{-} v_{\ell}\right) \sim \mathcal{O}\left(10^{-10}\right), \mathcal{B}\left(D_{s}^{*-} \rightarrow \ell^{-} v_{\ell}\right) \sim \mathcal{O}\left(10^{-6}\right)$ and $\mathcal{B}\left(B_{c}^{*-} \rightarrow \ell^{-} \nu_{\ell}\right) \sim \mathcal{O}\left(10^{-6}\right)$. Inspired by the potential prospects of LHCb, Belle-II, STCF, CEPC and FCC-ee experiments, we discussed the probabilities of experimental investigation on these purely leptonic decays. It is found that the measurements of these decays might be possible and feasible with the improvement of data statistics, analytical technique, and measurement precision in the future. (1) With the hadron-hadron collisions, the purely leptonic decays of $\rho^{-}, K^{*-}, D_{d, s}^{*-}$ and $B_{u, c}^{*-}$ mesons might be accessible at LHC experiments. (2) With the $e^{+} e^{-}$collisions, the purely leptonic decays of $D_{d, s}^{*-}$ and $B_{u, c}^{*-}$ mesons might be measurable with over $10^{12} Z^{0}$ bosons available at CEPC and FCC-ee experiments. In addition, the $D_{d, s}^{*-} \rightarrow \ell^{-} v_{\ell}$ decays could also be studied at Belle-II and SCTF experiments.
\end{abstract}

\section{Introduction}

In the quark model [1-3], mesons are generally regarded as bound states of the valence quark $q$ and antiquark $\bar{q}^{\prime}$. The classifications of mesons are usually based on the spinparity quantum number $J^{P}$ of the $q \bar{q}^{\prime}$ system. The spin $J$ of meson is given by the relation $|L-S| \leq J \leq|L+S|$. The orbital angular momentum and total spin of the $q \bar{q}^{\prime}$ sys-

\footnotetext{
a e-mail: yangyueling@htu.edu.cn (corresponding author)

b e-mail: sunjunfeng@htu.edu.cn (corresponding author)
}

tem are respectively $L$ and $S$, where $S=0$ for antiparallel quark spins, and $S=1$ for parallel quark spins. By convention, quarks have a positive parity and antiquarks have a negative parity. Hence, the parity of meson is $P=(-1)^{L+1}$. The $L=0$ states are the ground-state pseudoscalars with $J^{P}=0^{-}$and vectors with $J^{P}=1^{-}$. Both quarks and leptons are fermions with spin $S=1 / 2$. Mesons are composed of a pair of fermions - quark and antiquark, therefore, they could in principle decay into a pair of fermions, for example, lepton and antilepton. The experimental observation of the two-body purely leptonic decays of mesons could be a clear and characteristic manifestation of the quark model. These leptonic decays provide us with valuable opportunities to fully investigate the microstructure and properties of mesons. The study of two-body purely leptonic decays of mesons is very interesting and significant.

The valence quarks of the electrically charged mesons must have different flavors. Within the standard model (SM) of elementary particles, the purely leptonic decays of the charged mesons (PLDCM) are typically induced by the treelevel exchange of the gauge bosons $W$, the quanta of the weak interaction fields. Up to today, the masses of all the experimentally observed mesons are much less than those of $W$ bosons. Consequently, the massive $W$ bosons are virtual propagators rather than physical particles in the true picture of PLDCM. Phenomenologically, by integrating out the contributions from heavy dynamical degrees of freedom such as the $W$ fields, PLDCM can be properly described by the low-energy effective theory in analogy with the Fermi theory for $\beta$ decays. Considering the fact that leptons are free from the strong interactions, the corresponding effective Hamiltonian [4] for PLDCM could be written as the product of quark currents and leptonic currents,

$\mathcal{H}_{\text {eff }}=\frac{G_{F}}{\sqrt{2}} V_{q_{1} q_{2}}\left[\bar{q}_{1} \gamma_{\mu}\left(1-\gamma_{5}\right) q_{2}\right]\left[\bar{\ell} \gamma^{\mu}\left(1-\gamma_{5}\right) \nu_{\ell}\right]+$ h.c.

where the contributions of the $W$ bosons are embodied in the Fermi coupling constant $G_{F} \simeq 1.166 \times 10^{-5} \mathrm{GeV}^{-2}[1]$, 
and $V_{q_{1} q_{2}}$ is the Cabibbo-Kobayashi-Maskawa (CKM) [5,6] matrix element between the quarks in the charged mesons. The decay amplitudes can be written as,

$$
\begin{aligned}
\left\langle\ell \bar{v}_{\ell}\left|\mathcal{H}_{\mathrm{eff}}\right| M\right\rangle= & \frac{G_{F}}{\sqrt{2}} V_{q_{1} q_{2}}\left\langle\ell \bar{v}_{\ell}\left|\bar{\ell} \gamma^{\mu}\left(1-\gamma_{5}\right) \nu_{\ell}\right| 0\right\rangle \\
& \times\left\langle 0\left|\bar{q}_{1} \gamma_{\mu}\left(1-\gamma_{5}\right) q_{2}\right| M\right\rangle .
\end{aligned}
$$

The leptonic part of amplitudes can be calculated reliably with the perturbative theory. The hadronic matrix elements (HMEs) interpolating the diquark currents between the mesons concerned and the vacuum can be parameterized by the decay constants.

With the conventions of Refs. [7,8], the HMEs of diquark currents are defined as,

$$
\begin{aligned}
\left\langle 0\left|\bar{q}_{1}(0) \gamma_{\mu} q_{2}(0)\right| P(k)\right\rangle & =0, \\
\left\langle 0\left|\bar{q}_{1}(0) \gamma_{\mu} \gamma_{5} q_{2}(0)\right| P(k)\right\rangle & =i f_{P} k_{\mu}, \\
\left\langle 0\left|\bar{q}_{1}(0) \gamma_{\mu} q_{2}(0)\right| V(k, \epsilon)\right\rangle & =f_{V} m_{V} \epsilon_{\mu}, \\
\left\langle 0\left|\bar{q}_{1}(0) \gamma_{\mu} \gamma_{5} q_{2}(0)\right| V(k, \epsilon)\right\rangle & =0,
\end{aligned}
$$

where the nonperturbative parameters of $f_{P}$ and $f_{V}$ are the decay constants of pseudoscalar $P$ and vector $V$ mesons, respectively; and $m_{V}$ and $\epsilon_{\mu}$ are the mass and polarization vector, respectively. To the lowest order, the decay widths are written as,

$$
\begin{aligned}
& \Gamma\left(P \rightarrow \ell \bar{v}_{\ell}\right)=\frac{G_{F}^{2}}{8 \pi}\left|V_{q_{1} q_{2}}\right|^{2} f_{P}^{2} m_{P} m_{\ell}^{2}\left(1-\frac{m_{\ell}^{2}}{m_{P}^{2}}\right)^{2}, \\
& \Gamma\left(V \rightarrow \ell \bar{v}_{\ell}\right)=\frac{G_{F}^{2}}{12 \pi}\left|V_{q_{1} q_{2}}\right|^{2} f_{V}^{2} m_{V}^{3}\left(1-\frac{m_{\ell}^{2}}{m_{V}^{2}}\right)^{2}\left(1+\frac{m_{\ell}^{2}}{2 m_{V}^{2}}\right),
\end{aligned}
$$

where $m_{P}$ and $m_{\ell}$ are the masses of the charged pseudoscalar meson and lepton, respectively.

It is clearly seen from the above formula that the highly precise measurements of PLDCM will allow the relatively accurate determinations of the product of the decay constants and CKM elements, $\left|V_{q_{1} q_{2}}\right| f_{P, V}$. Theoretically, the decay constants are nonperturbative parameters, and they are closely related with the $\bar{q}_{1} q_{2}$ wave functions at the origin which cannot be computed from first principles. There still exist some discrepancies among theoretical results of the decay constants with different methods, such as the potential model, QCD sum rules, lattice QCD, and so on. If the magnitudes of CKM element $\left|V_{q_{1} q_{2}}\right|$ are fixed to the values of Ref. [1], the decay constants $f_{P, V}$ will be experimentally measured, and be used to seriously examine the different calculations on the decay constants with various theoretical models. Likewise, if the decay constants $f_{P, V}$ are well known to sufficient precision, the magnitudes of the corresponding CKM element will be experimentally determined, and provide complementary information to those from other processes. Within SM, the $P \rightarrow \ell \bar{v}_{\ell}$ and $V \rightarrow \ell \bar{v}_{\ell}$ decays are induced by the axial-vector current of Eq. (4) and vector current of Eq. (5), respectively; and the electroweak interactions assign the vector-minus-axial-vector $(V-A)$ currents to the $W$ bosons. The CKM elements determined from two different and complementary parts of the electroweak interactions, charged vector and axial-vector currents, could be independently examined. The latest CKM elements determined by PLDCM, such as $\left|V_{u s}\right|,\left|V_{c d}\right|$ and $\left|V_{c s}\right|$, differ somewhat from those by exclusive and inclusive semileptonic meson decays [1]. The CKM elements extracted from various processes can be combined to test the electroweak characteristic chargedcurrent $V-A$ interactions.

Within SM, the lepton-gauge-boson electroweak gauge couplings are generally believed to be universal and process independent, which is called lepton flavor universality (LFU). However, there are some hints of LFU discrepancies between SM predictions and experimental measurements, such as the ratios of branching fractions of semileptonic $B$ decays $R\left(D^{(*)}\right) \equiv \mathcal{B}\left(\bar{B} \rightarrow D^{(*)} \tau \bar{\nu}_{\tau}\right) / \mathcal{B}\left(\bar{B} \rightarrow D^{(*)} \ell \bar{\nu}_{\ell}\right)$ with $\ell$ $=e / \mu[1]$. The LFU validity can be carefully investigated through the PLDCM processes. Beyond SM, some possible new heavy particles accompanied with novel interactions, such as the charged higgs bosons, would affect PLDCM and LFU, and might lead to detectable effects. So PLDCM provide good arenas to search for the smoking gun of new physics (NP) beyond SM.

By considering the angular momentum conservation and the final states including a left-handed neutrino or righthanded antineutrino, the purely leptonic decay width of charged pseudoscalar meson, Eq. (7), is proportional to the square of the lepton mass. This is called helicity suppression. While there is no helicity suppression for the purely leptonic decay of charged vector meson (PLDCV). From the analytical expressions of Eqs. (7) and (8), the decay width of pseudoscalar meson is suppressed by the factor $m_{\ell}^{2} / m_{P}^{2}$ compared with that of vector meson. What's more, both the masses and the decay constants of vector mesons are relatively larger than those of corresponding pseudoscalar mesons, which would result in an enhancement of the decay widths for vector mesons. Of course, the vector mesons decay dominantly through the strong and/or electromagnetic interactions. The branching ratios for the PLDCV weak decays are usually very small, sometimes even close to the accessible limits of the existing and the coming experiments.

Inspired by the potential prospects of the future highintensity and high-energy frontiers, along with the noticeable increase of experimental data statistics, the remarkable improvement of analytical technique and the continuous enhancement of measurement precision, the carefully experimental study of PLDCV might be possible and feasible. In this paper, we will focus on the PLDCV within SM to just provide a ready reference. The review of the purely leptonic 
decays of charged pseudoscalar mesons can be found in Ref. [1].

\section{$2 \rho^{-} \rightarrow \ell^{-} \bar{v}_{\ell}$ decays}

The mass of the $\rho^{ \pm}$meson, $m_{\rho}=775.11(34) \mathrm{MeV}$ [1], is much larger than that of two-pion pair. The rate of the $\rho$ meson decay into two pions via the strong interactions is almost $100 \%$, which results in the very short lifetime $\tau_{\rho} \sim$ $4.4 \times 10^{-24} \mathrm{~s}$ [1]. The direct measurements of the electroweak properties of the $\rho$ meson would definitely be very challenging. It is evident from Eq. (8) that the parameter of $\left|V_{u d}\right| f_{\rho}$ could be experimentally determined from the observations of decay widths for the $\rho^{-} \rightarrow \ell^{-} \bar{v}_{\ell}$ decays (if it is not specified, the corresponding charge-conjugation processes are included in this paper), with the coupling constant $G_{F}$, the masses of lepton $m_{\ell}$ and meson $m_{\rho}$.

The precise values of the CKM element $\left|V_{u d}\right|$ in ascending order of measurement accuracy mainly come from $\beta$ transitions between the super-allowed nuclear analog states with quantum number of both $J^{P}=0^{+}$and isospin $I=1$, between mirror nuclei with $I=1 / 2$, between neutron and proton, between charged and neutral pions [9]. These four results for $\left|V_{u d}\right|$ are basically consistent with one another. The result of the super-allowed $0^{+} \rightarrow 0^{+}$nuclear $\beta$ transitions has an uncertainty a factor of about 10 smaller than the other results, and thus dominates the weighted average value [9]. The best value from super-allowed nuclear $\beta$ transitions is $\left|V_{u d}\right|=$ $0.97370(14)$ [1], which is smaller compared with the 2018 value $\left|V_{u d}\right|=0.97420(21)[10]$, as illustrated Fig. 1. This reduction of the value of $\left|V_{u d}\right|$ leads to a slight deviation from the first row unitarity requirement $\left|V_{u d}\right|^{2}+\left|V_{u s}\right|^{2}+$ $\left|V_{u b}\right|^{2}=1$. The current precision of the CKM element $\left|V_{u d}\right|$ is about $0.01 \%$. The latest value from the global fit in SM, $\left|V_{u d}\right|=0.97401$ (11) [1], will be used in our calculation.

The decay constant $f_{\rho}$ is an very important characteristics of the $\rho$ meson. Compared with the CKM element $\left|V_{u d}\right|$, the present precision of decay constant $f_{\rho}$ is still not very high and needs to be improved. Theoretically, the estimations from different methods are more or less different from each other and even calculations with the same method sometimes give the diverse results. Some theoretical estimations on the decay constant $f_{\rho}$ are presented in Table 1. Experimentally, the decay constant $f_{\rho}$ can be obtained from the 1-prong hadronic $\tau^{ \pm} \rightarrow \rho^{ \pm} \nu_{\tau}$ decay. The partial width for the $\tau \rightarrow V \nu_{\tau}$ decay is given by Ref. [37],

$\Gamma(\tau \rightarrow V \nu)=\mathcal{S} \frac{G_{F}^{2} m_{\tau}^{3}}{16 \pi}\left|V_{q_{1} q_{2}}\right|^{2} f_{V}^{2}\left(1-\frac{m_{V}^{2}}{m_{\tau}^{2}}\right)^{2}\left(1+\frac{2 m_{V}^{2}}{m_{\tau}^{2}}\right)$,

where the factor $\mathcal{S}=1.0154$ includes the electroweak corrections [37-39]. With the mass $m_{\tau}=1776.86(12) \mathrm{MeV}$ and

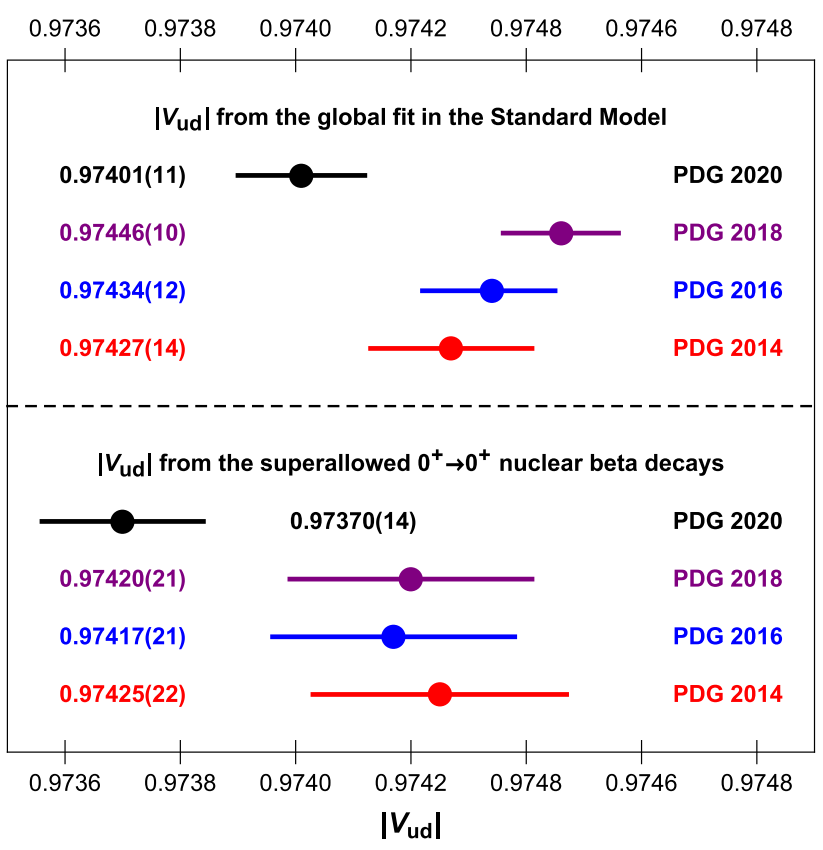

Fig. 1 The values of the CKM element $\left|V_{u d}\right|$ from Particle Data Group (PDG)

lifetime $\tau_{\tau}=290.3(5)$ fs [1], and branching ratio $\mathcal{B}(\tau \rightarrow \rho \nu)$ $=25.19(33) \%$ [1], one can easily extract the decay constant $f_{\rho}^{\exp }=207.7 \pm 1.6 \mathrm{MeV}$, which agrees well with the latest numerical simulation result from lattice QCD $f_{\rho}=$ $208.5 \pm 5.5 \pm 0.9 \mathrm{MeV}$ [34]. The more accurate decay constant $f_{\rho}^{\exp }$ will be used in our calculation.

For the $\rho^{-} \rightarrow \ell^{-} \bar{v}_{\ell}$ decays, one can obtain the PLDCV partial decay widths with Eq. (8) and the corresponding branching ratios with the full width $\Gamma_{\rho}=149.1 \pm 0.8 \mathrm{MeV}$ [1],

$$
\begin{aligned}
\Gamma\left(\rho^{-} \rightarrow e^{-} \bar{v}_{e}\right) & =68.8 \pm 1.2 \mu \mathrm{eV}, \\
\Gamma\left(\rho^{-} \rightarrow \mu^{-} \bar{v}_{\mu}\right) & =66.9 \pm 1.1 \mu \mathrm{eV}, \\
\mathcal{B}\left(\rho^{-} \rightarrow e^{-} \bar{v}_{e}\right) & =(4.6 \pm 0.1) \times 10^{-13}, \\
\mathcal{B}\left(\rho^{-} \rightarrow \mu^{-} \bar{v}_{\mu}\right) & =(4.5 \pm 0.1) \times 10^{-13},
\end{aligned}
$$

where the uncertainties come from the uncertainties of mass $m_{\rho}$, decay constant $f_{\rho}$ and CKM element $\left|V_{u d}\right|$, and additional decay width $\Gamma_{\rho}$ for branching ratios. Clearly, the branching ratios are very small. Given the identification efficiency and pollution from background, the $\rho^{-} \rightarrow \ell^{-} \bar{v}_{\ell}$ decays might be measured only with more than $10^{14} \rho^{ \pm}$ events available.

There are at least three possible ways to experimentally produce the charged $\rho$ mesons in the electron-position collisions, (a) the prompt pair production $e^{+} e^{-} \rightarrow \rho^{+} \rho^{-}$, (b) the pair production via $V$ decay $1^{--} \rightarrow \rho^{+} \rho^{-}$, and (c) the single production via $V$ decay $1^{--} \rightarrow \rho^{ \pm} h^{\mp}$. The cross section $\sigma\left(e^{+} e^{-} \rightarrow \rho^{+} \rho^{-}\right)$has been determined by the BaBar group to be $19.5 \pm 1.6 \pm 3.2 \mathrm{fb}$ near the center-of-mass energy $\sqrt{s}$ 
Table 1 Decay constant $f_{\rho}$ (in the unit of $\mathrm{MeV}$ ) obtained from the diffractive vector meson production using deep inelastic scattering (DVP), QCD sum rules (SR), relativistic quark model (RQM), light-front quark model (LFQM), lattice QCD (LQCD) and so on

\begin{tabular}{llllll}
\hline DVP & $143[11]^{\mathrm{a}}$ & $147[11]^{\mathrm{b}}$ & $153[11]^{\mathrm{c}}$ & $161[11]^{\mathrm{d}}$ & $211[14]$ \\
SR & $201 \pm 5[15]^{\mathrm{e}}$ & $205 \pm 10[15]^{\mathrm{f}}$ & $194[15]^{\mathrm{g}}$ & $198 \pm 7[18]$ & $206 \pm 7[19]$ \\
RQM & $168.3[20]^{\mathrm{h}}$ & $151.3[20]^{\mathrm{i}}$ & $190.1[20]^{\mathrm{j}}$ & $175.4[20]^{\mathrm{k}}$ & $219[22]$ \\
LFQM & $246[23]^{\mathrm{l}}$ & $215[23]^{\mathrm{m}}$ & $205[24]^{l}$ & $166_{-4}^{+2}[25]^{\mathrm{n}}$ & $210 \pm 6[25]^{\mathrm{o}}$ \\
LFQM & $215 \pm 5[26]$ & $211 \pm 1[27]$ & $242_{-24}^{+23}[28]$ & & \\
LQCD & $239.4 \pm 7.3[29,30]$ & $210 \pm 15[31]$ & $239 \pm 18[32]$ & $199 \pm 4[33]$ & $208.5 \pm 5.6[34]$ \\
Other & $490[22]$ & $207[35]$ & $254[36]$ & & \\
\hline
\end{tabular}

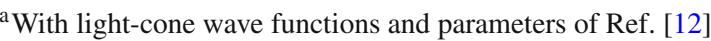

${ }^{b}$ With light-cone wave functions and parameters of Ref. [13]

${ }^{\mathrm{c}}$ With Gaussian wave functions and parameters of Ref. [12]. ${ }^{\mathrm{d}}$ With Gaussian wave functions and parameters of Ref. [13]

${ }^{\mathrm{e}}$ With nonlocal condensates like functions. ${ }^{\mathrm{f}}$ With Ball-Braun wave functions [16]

gWith Chernyak-Zhitnitsky wave functions [17]

${ }^{\mathrm{h}}$ With Gaussian spatial wave functions and adjusted parameters of Ref. [20]

${ }^{i}$ With Gaussian spatial wave functions and parameters of Ref. [21]

jWith rational spatial wave functions and adjusted parameters of Ref. [20]

${ }^{\mathrm{k}}$ With rational spatial wave functions and parameters of Ref. [21]

${ }^{1}$ With Coulomb plus linear potential model

${ }^{\mathrm{m}}$ With Coulomb plus harmonic oscillator potential model

${ }^{\mathrm{n}}$ With a dilation parameter $\kappa=0.54 \mathrm{GeV}$

${ }^{\circ}$ With a dilation parameter $\kappa=0.68 \mathrm{GeV}$
$=10.58 \mathrm{GeV}$ [40]. Assuming the production cross section $\sigma$ $\propto 1 / s[41,42]$, it could be speculated that $\sigma\left(e^{+} e^{-} \rightarrow \rho^{+} \rho^{-}\right)$ $\sim 230 \mathrm{fb}$ near $\sqrt{s}=3.1 \mathrm{GeV}$. There would be only about $10^{6} \rho^{+} \rho^{-}$pairs with a data sample of $50 \mathrm{ab}^{-1}[43,44]$ near $\sqrt{s} \approx m_{\Upsilon(4 S)}$ at the Belle-II detector or a data sample of 10 $\mathrm{ab}^{-1}$ [45] near $\sqrt{s} \approx m_{J / \psi}$ with the future super-tau-charm factory like STCF or SCTF [46-48]. The charge $\rho$ mesons can in principle be produced from the $\Upsilon(4 S), J / \psi$ and $\phi$ decays. The branching ratios are

$$
\begin{aligned}
\mathcal{B}\left(\Upsilon(4 S) \rightarrow \rho^{+} \rho^{-}\right) & <5.7 \times 10^{-6}[39], \\
\mathcal{B}\left(J / \psi \rightarrow \rho^{+} \rho^{-}\right) & \sim 10^{-3}[1,47], \\
\mathcal{B}\left(J / \psi \rightarrow \rho^{ \pm} \pi^{\mp}\right) & \sim 10^{-2}[1], \\
\mathcal{B}\left(\phi \rightarrow \rho^{ \pm} \pi^{\mp}\right) & \sim 10^{-1}[1],
\end{aligned}
$$

where the branching ratio $\mathcal{B}\left(J / \psi \rightarrow \rho^{+} \rho^{-}\right)$is assumed to be the same order of magnitude as $\mathcal{B}\left(J / \psi \rightarrow K^{*+} K^{*-}\right) \sim$ $10^{-3}$ [1] from the phenomenological analysis based on the flavor-SU (3) symmetry [49]. Now, there are $7.7 \times 10^{8} \Upsilon(4 S)$ events at Belle [50], $10^{10} \mathrm{~J} / \psi$ events [46] at BES-III, and $2.4 \times 10^{10} \phi$ events at KLOE/KLOE-2 [51] available. It is expected that only about $5 \times 10^{10} \Upsilon(4 S)$ events $[43,44]$ and $10^{13} \mathrm{~J} / \psi$ events at SCTF or STCF [46] could be accumulated. It is clearly seen that unless a very significant enhancement to branching ratios from some NP, the experimental data on the $\rho^{ \pm}$meson are too scarce to search for the $\rho^{-}$ $\rightarrow \ell^{-} \bar{v}_{\ell}$ decays at the electron-position collisions in the near future, which result in the natural difficulties to understand the $\rho$ meson.

The production cross sections of prompt $J / \psi$ and $J / \psi$ from-b mesons in proton-proton collisions at $\sqrt{s}=13$
$\mathrm{TeV}$ are measured by $\mathrm{LHCb}$ to be $15.0 \pm 0.6 \pm 0.7 \mu \mathrm{b}$ and $2.25 \pm 0.09 \pm 0.10 \mu \mathrm{b}$, respectively, [52,53]. It is expected that some $10^{12} \mathrm{~J} / \psi$ events could be accumulated at $\sqrt{\mathrm{s}}=$ $13 \mathrm{TeV}$ with an integrated luminosity of $300 \mathrm{fb}^{-1}$ at LHCb [54]. There are only about $10^{10} \rho^{ \pm}$mesons from $J / \psi$ decays available for prying into the $\rho^{ \pm}$PLDCV decays. At the same time, the inclusive cross-sections for prompt charm production at $\mathrm{LHCb}$ at $\sqrt{s}=13 \mathrm{TeV}$ are measured to be $\mathcal{O}(1 \mathrm{mb})$ [55-57]. Analogically assuming the inclusive cross section of prompt $\rho^{ \pm}$meson production at LHCb at $\sqrt{s}=13 \mathrm{TeV}$ is $\mathcal{O}(10 \mathrm{mb})$, some $3 \times 10^{15} \rho^{ \pm}$events would be accumulated with an integrated luminosity of $300 \mathrm{fb}^{-1}$ at $\mathrm{LHCb}$ [54]. Optimistically assuming the reconstruction efficiency is about $10 \%$, there would be about $\mathcal{O}\left(10^{2}\right)$ events of the $\rho^{-}$ $\rightarrow \ell^{-} \bar{\nu}_{\ell}$ decays at $\mathrm{LHCb}$, and more events with the enhanced branching ratios from NP contributions. Even through it will be very challenging for experimental analysis due to the complex background in hadron-hadron collisions, there is still a strong presumption that the $\rho^{-} \rightarrow \ell^{-} \bar{v}_{\ell}$ decays could be explored and studied at LHC in the future. In addition, it is expected that an integrated luminosity exceeding $10 \mathrm{ab}^{-1}$ would be reached at the future HE-LHC experiments [58]. More experimental data at HE-LHC would make the study of the $\rho^{-} \rightarrow \ell^{-} \bar{v}_{\ell}$ decays indeed feasible in hadron-hadron collisions.

\section{$3 \mathrm{~K}^{*-} \rightarrow \ell^{-} \overline{\boldsymbol{v}}_{\ell}$ decays}

The parameter product $\left|V_{u s}\right| f_{K^{*}}$ could be experimentally determined from the $K^{*-} \rightarrow \ell^{-} \bar{v}_{\ell}$ decays using Eq. (8). 
Like the $\rho^{ \pm}$meson, the mass of the $K^{* \pm}$ meson, $m_{K^{* \pm}}=$ 895.5(8) MeV, is above the threshold of $K \pi$ pair, and the partial branching ratio of the $K^{*}$ meson decay into $K \pi$ pair via the strong interactions is almost $100 \%$ [1]. It is not hard to imagine that the very short lifetime $\tau_{K^{*}} \sim 1.4 \times 10^{-23} \mathrm{~s}$ would enable the measurements of the electroweak properties of the $K^{*}$ meson to be very challenging or nearly impossible.

The CKM element $\left|V_{u s}\right| \simeq \lambda$ up to the order of $\mathcal{O}\left(\lambda^{6}\right)$, where $\lambda$ is a Wolfenstein parameter. The current precision of the CKM element $\left|V_{u s}\right|$ from purely leptonic and semileptonic $K$ meson decays and hadronic $\tau$ decays are $0.2 \%, 0.3 \%$ and $0.6 \%$, respectively. It is seen from Fig. 2 that these three results for $\left|V_{u s}\right|$ are not very consistent with one another. So if the $K^{*-} \rightarrow \ell^{-} \bar{v}_{\ell}$ decays could be measured, they would provide another determination and constraint to $\left|V_{u s}\right|$. Probably due to the reduction of the value of $\left|V_{u d}\right|$, the latest value from the global fit in SM, $\left|V_{u s}\right|=0.22650$ (48) [1], is slightly larger than the 2018 value, to satisfy the first row unitarity requirement.

Some theoretical results on the decay constant $f_{K^{*}}$ are presented in Table 2. Like the case of the decay constant $f_{\rho}$, the model dependence of theoretical estimations on the decay constant $f_{K^{*}}$ is also obvious. Experimentally, the decay constant $f_{K^{*}}$ can be obtained from the hadronic $\tau^{ \pm} \rightarrow K^{* \pm} \nu_{\tau}$ decays. Using Eq. (9) and experimental data on branching ratio $\mathcal{B}\left(\tau \rightarrow K^{*} \nu\right)=1.20(7) \%$ [1], one can obtain the decay constant $f_{K^{*}}^{\text {exp }}=202.5_{-6.7}^{+6.5} \mathrm{MeV}$. The value of $f_{K^{*}}^{\text {exp }}$ is much less than that of LQCD results, and will be used in our calculation.

For the $K^{*-} \rightarrow \ell^{-} \bar{\nu}_{\ell}$ decays, the SM expectations on the partial decay widths and branching ratios are,

$\Gamma\left(K^{*-} \rightarrow e^{-} \bar{v}_{e}\right)=5.5 \pm 0.4 \mu \mathrm{eV}$,

$\Gamma\left(K^{*-} \rightarrow \mu^{-} \bar{v}_{\mu}\right)=5.3 \pm 0.4 \mu \mathrm{eV}$,

$\mathcal{B}\left(K^{*-} \rightarrow e^{-} \bar{v}_{e}\right)=(1.2 \pm 0.1) \times 10^{-13}$,

$\mathcal{B}\left(K^{*-} \rightarrow \mu^{-} \bar{v}_{\mu}\right)=(1.2 \pm 0.1) \times 10^{-13}$.

The decay width $\Gamma_{K^{*}}=46.2 \pm 1.3 \mathrm{MeV}$ [1] is used in our calculation. It is apparent that more than $10^{14} \mathrm{~K}^{* \pm}$ events are the minimum requirement for experimentally studying the $K^{*-} \rightarrow \ell^{-} \bar{v}_{\ell}$ decays.

Based on the $U$-spin symmetry, the production mechanism of the $K^{* \pm}$ mesons in electron-position collisions is similar to that of the $\rho^{ \pm}$mesons. An educated guess is that the cross section $\sigma\left(e^{+} e^{-} \rightarrow K^{*+} K^{*-}\right) \sim 20 \mathrm{fb}$ and $230 \mathrm{fb}$ near $\sqrt{s} \sim$ $m_{\Upsilon(4 S)}$ and $m_{J / \psi}$, respectively. The branching ratios of $J / \psi$ decays are [1],

$$
\begin{aligned}
\mathcal{B}\left(J / \psi \rightarrow K^{*+} K^{*-}\right) & =\left(1.00_{-0.40}^{+0.22}\right) \times 10^{-3}, \\
\mathcal{B}\left(J / \psi \rightarrow K^{* \pm} K^{\mp}\right) & =\left(6.0_{-1.0}^{+0.8}\right) \times 10^{-3} \\
\mathcal{B}\left(J / \psi \rightarrow K^{* \pm} K^{\mp} \pi^{0}\right) & =(4.1 \pm 1.3) \times 10^{-3} \\
\mathcal{B}\left(J / \psi \rightarrow K^{* \pm} K_{S}^{0} \pi^{\mp}\right) & =(2.0 \pm 0.5) \times 10^{-3},
\end{aligned}
$$

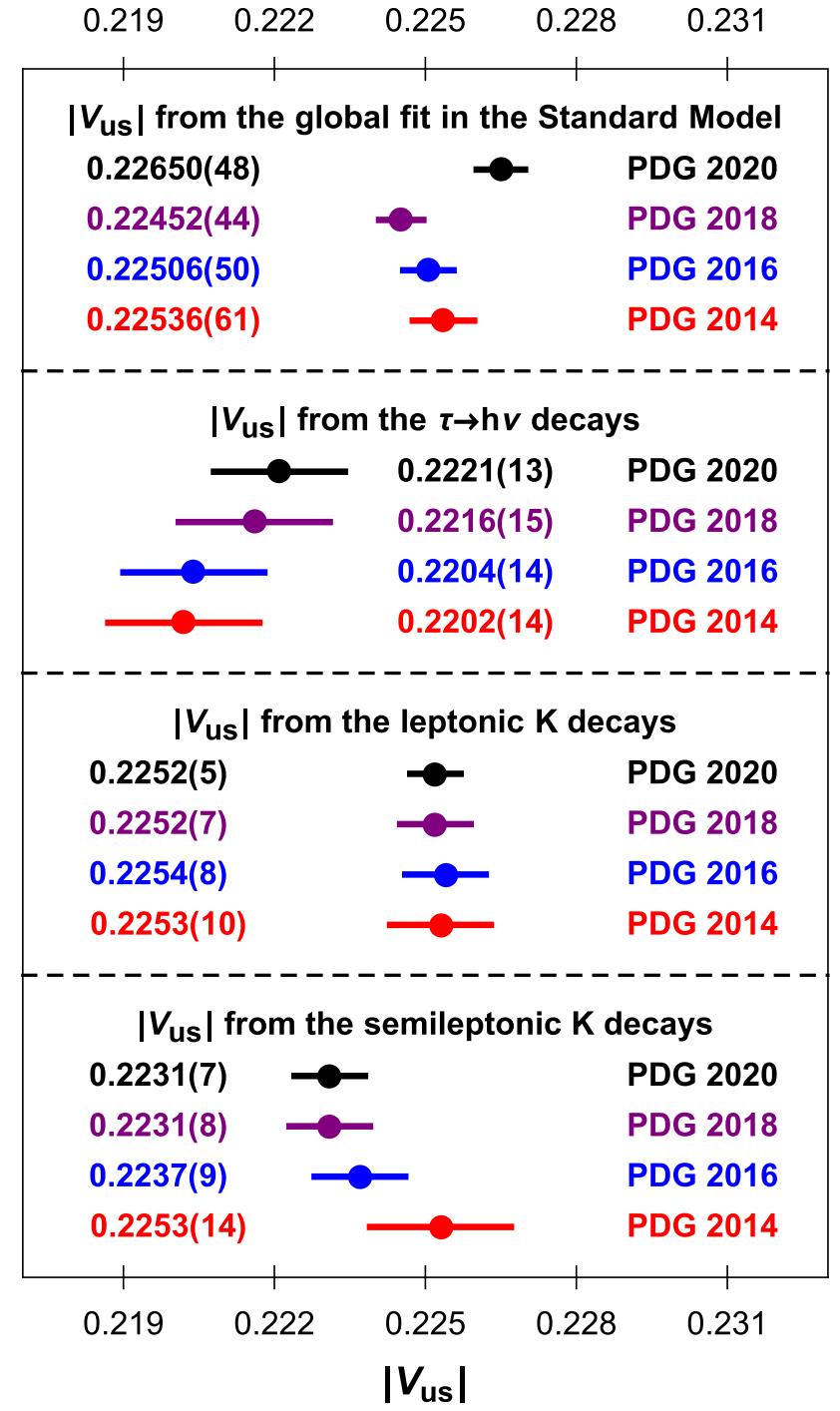

Fig. 2 The values of the CKM element $\left|V_{u s}\right|$ from PDG

$$
\begin{aligned}
\mathcal{B}\left(J / \psi \rightarrow K^{* \pm} K_{2}^{*}(1430)^{\mp}\right) & =(3.4 \pm 2.9) \times 10^{-3}, \\
\mathcal{B}\left(J / \psi \rightarrow K^{* \pm} K^{*}(700)^{\mp}\right) & =\left(1.0_{-0.6}^{+1.0}\right) \times 10^{-3} .
\end{aligned}
$$

It is approximately estimated that $\mathcal{B}\left(J / \psi \rightarrow K^{* \pm} X^{\mp}\right) \sim$ $1.8 \%$. Hence, the experimental data on the $K^{* \pm}$ mesons at the $e^{+} e^{-}$collisions, which would be available by either the prompt $K^{*+} K^{*-}$ pair production at SuperKEKB and SCTF experiments or the production via $10^{13} \mathrm{~J} / \psi$ decay at SCTF, are far from sufficient for investigating the $\mathrm{K}^{*-} \rightarrow$ $\ell^{-} \bar{v}_{\ell}$ decays. If we assume that the inclusive cross section of prompt $K^{* \pm}$ meson production in $p p$ collisions at the centerof-mass energy of $13 \mathrm{TeV}$ is similar to that of $\rho^{ \pm}$mesons, about $\mathcal{O}(10 \mathrm{mb})$, there would be some $3 \times 10^{15} \mathrm{~K}^{* \pm}$ events to be available with an integrated luminosity of $300 \mathrm{fb}^{-1}$ at $\mathrm{LHCb}$, which correspond to about $\mathcal{O}\left(10^{2}\right)$ events of the $K^{*-} \rightarrow \ell^{-} \bar{v}_{\ell}$ decays. It should be some glimmer of hope for observation and scrutinies of the $K^{*-} \rightarrow \ell^{-} \bar{v}_{\ell}$ decays 
Table 2 The theoretical values of decay constant $f_{K^{*}}$ (in the unit of $\mathrm{MeV}$ ), where the legends are the same as those in Table 1

\begin{tabular}{llllll}
\hline RQM & $170.5[20]^{\mathrm{a}}$ & $153.1[20]^{\mathrm{b}}$ & $192.6[20]^{\mathrm{c}}$ & $177.6[20]^{\mathrm{d}}$ & $236[22]$ \\
LFQM & $256[23]^{\mathrm{e}}$ & $223[23]^{\mathrm{f}}$ & $224[24]^{\mathrm{e}}$ & $186_{-3}^{+2}[25]^{\mathrm{g}}$ & $204_{-9}^{+7}[25]^{\mathrm{h}}$ \\
LFQM & $217 \pm 5[26]$ & $223 \pm 1[27]$ & $253 \pm 25[28]$ & \\
LQCD & $255.5 \pm 6.5[29,30]^{\mathrm{i}}$ & $257.7 \pm 6.4[29,30]^{\mathrm{j}}$ & $240 \pm 18[59]$ & \\
other & $226 \pm 28[18]$ & $508[22]$ & $241[35]$ & \\
\hline
\end{tabular}

${ }^{a}$ With Gaussian spatial wave functions and adjusted parameters of Ref. [20]

${ }^{b}$ With Gaussian spatial wave functions and parameters of Ref. [21]

${ }^{c}$ With rational spatial wave functions and adjusted parameters of Ref. [20]

${ }^{\mathrm{d}}$ With rational spatial wave functions and parameters of Ref. [21]

${ }^{\mathrm{e}}$ With Coulomb plus linear potential model

${ }^{\mathrm{f}}$ With Coulomb plus harmonic oscillator potential model

${ }^{\mathrm{g}}$ With a dilation parameter $\kappa=0.54 \mathrm{GeV}$

${ }^{\text {h}}$ With a dilation parameter $\kappa=0.68 \mathrm{GeV}$

iThe $K$ meson mass is used as input

${ }^{\mathrm{j}}$ The $\phi$ meson mass is used as input at hadron-hadron collisions in the future, particularly at the planning HE-HLC.

\section{$4 D_{d}^{*-} \rightarrow \ell^{-} \bar{v}_{\ell}$ decays}

The mass of $D_{d}^{*}$ mesons, $m_{D_{d}^{*}}=2010.26(5) \mathrm{MeV}$, are just above the threshold of $D \pi$ pair. The $D_{d}^{*}$ meson decays via the strong interactions are dominant, and the ratio of branching ratios [1], $\mathcal{B}\left(D_{d}^{* \pm} \rightarrow D_{d}^{ \pm} \pi^{0}\right) / \mathcal{B}\left(D_{d}^{* \pm} \rightarrow D_{u} \pi^{ \pm}\right)=$ $30.7(5) \% / 67.7(5) \% \sim 1 / 2$, basically agrees with the relations of isospin symmetry. It should be pointed out that the $D_{d}^{*}$ strong decays are highly suppressed by the compact phase spaces because of $m_{D_{d}^{*}}-m_{D}-m_{\pi}<6 \mathrm{MeV}$. The branching ratio of the magnetic dipole transition is small, $\mathcal{B}\left(D_{d}^{*} \rightarrow D_{d} \gamma\right)$ $=1.6(4) \%$ [1]. Hence, the decay width of $D_{d}^{*}$ mesons is narrow, $\Gamma_{D_{d}^{*}}=83.4 \pm 1.8 \mathrm{keV}$ [1]. From the $D_{d}^{*-} \rightarrow \ell^{-} \bar{v}_{\ell}$ decays, the parameter $\left|V_{c d}\right| f_{D_{d}^{*}}$ is expected to be experimentally determined.

Currently, the precise values of the CKM element $\left|V_{c d}\right|$ comes mainly from the leptonic and semileptonic $D$ meson decays [1], as illustrated in Fig. 3. Because of the decay width of Eq. (7) being proportional to $m_{\ell}^{2}$, the $D^{-} \rightarrow e^{-} \bar{v}_{e}$ decay is helicity suppressed. And the $D^{-} \rightarrow \tau^{-} \bar{v}_{\tau}$ decay suffers from the complications caused by the additional neutrino in $\tau$ decays. The $D^{-} \rightarrow \mu^{-} \bar{v}_{\mu}$ decay is the most favorable mode for experimental measurement. For the values of $\left|V_{c d}\right|$ from the purely leptonic decay $D^{-} \rightarrow \mu^{-} \bar{v}$, the experimentally statistical uncertainties are dominant uncertainties. For the values of $\left|V_{c d}\right|$ from the semileptonic $D$ meson decays, the theoretical uncertainties from the form factor controlled by nonperturbative dynamics are dominant uncertainties. It is clearly seen from Fig. 3 that the experimental uncertainties have not decreased significantly recently. Besides, $\left|V_{c d}\right|$ can also be determined from the neutrino-induced charm production data [1], but the relevant experimental data have not been updated after the measurements given by the CHARM-

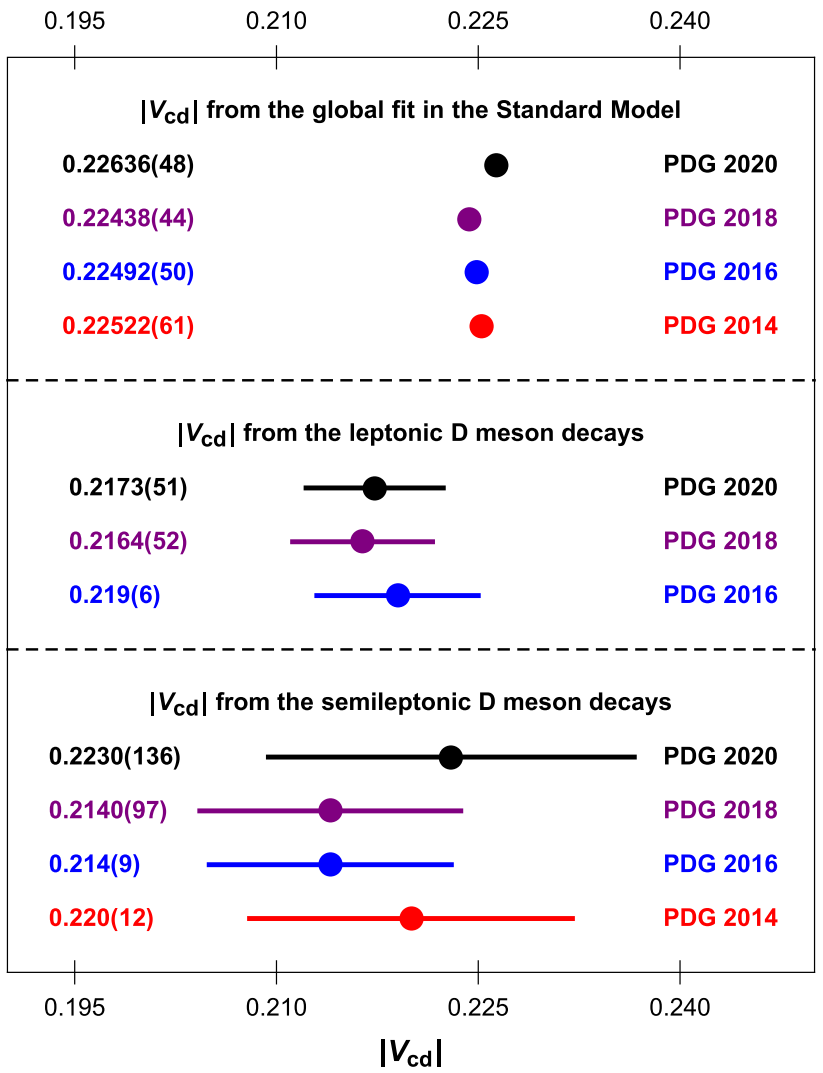

Fig. 3 The values of the CKM element $\left|V_{c d}\right|$ from PDG

II Collaboration in 1999 [60]. According to the Wolfenstein parameterization of the CKM matrix, there is an approximate relation between its elements $\left|V_{c d}\right|=\left|V_{u s}\right|=\lambda$ up to $\mathcal{O}\left(\lambda^{4}\right)$. However, the measurement precision of the CKM element $\left|V_{c d}\right|$ from both leptonic and semileptonic $D$ meson decays is generally about an order of magnitude smaller than that of $\left|V_{u s}\right|$ from leptonic and semileptonic $K$ meson decays for the moment. The most precise values are from the global fit in $\mathrm{SM},\left|V_{c d}\right|=0.22636(48)$ [1] with uncertainties $\sim 0.2 \%$. 
Table 3 The theoretical values of decay constant $f_{D_{d}^{*}}$ (in the unit of MeV), where the legends are the same as those in Table 1, NRQM is an abbreviation for nonrelativistic quark model

\begin{tabular}{|c|c|c|c|c|c|}
\hline NRQM & $223_{-19}^{+23}[61]$ & $307[62]^{\mathrm{a}}$ & $253[62]^{\mathrm{b}}$ & $353.8[63]^{\mathrm{a}}$ & $290.3[63]^{\mathrm{b}}$ \\
\hline NRQM & 391 [22] & $290[64]^{\mathrm{a}}$ & $210[64]^{\mathrm{b}}$ & $332[65,66]$ & \\
\hline RQM & $310[22]$ & $315[65,66]$ & $327 \pm 13[67]^{\mathrm{c}}$ & $252 \pm 10[67]^{\mathrm{d}}$ & \\
\hline LFQM & $254[68]^{\mathrm{e}}$ & $228[68]^{\mathrm{f}}$ & $259.6 \pm 14.6[69]^{\mathrm{g}}$ & $306.3_{-17.7}^{+18.2}[69]^{\mathrm{h}}$ & $253 \pm 7[27]$ \\
\hline LFQM & $230[24]^{\mathrm{i}}$ & $226.6_{-10.2}^{+5.9}[25]^{\mathrm{j}}$ & $230.1 \pm 6.2[25]^{\mathrm{k}}$ & $245_{-34}^{+35}[26]$ & $230 \pm 29[28]$ \\
\hline LFQM & $252.0_{-11.6}^{+13.8}[70]^{\mathrm{g}}$ & $264.9_{-9.5}^{+10.2}[70]^{\mathrm{h}}$ & $272[71]^{1}$ & $260[71]^{\mathrm{m}}$ & $269[71]^{\mathrm{n}}$ \\
\hline LQCD & $245 \pm 20[75]$ & $234 \pm 26[76]^{\circ}$ & $278 \pm 16[77]$ & $223.5 \pm 8.7[78]$ & 234(6) [79] \\
\hline SR & $242_{-12}^{+20}[80-82]$ & $263 \pm 21[83]$ & $252.2 \pm 22.7[84]$ & $250 \pm 11[85]$ & \\
\hline other & $186[71]^{\mathrm{p}}$ & $273 \pm 13[86]$ & $341 \pm 23[87]$ & $237[88]$ & \\
\hline
\end{tabular}

${ }^{a}$ Without QCD radiative corrections.

${ }^{\mathrm{b}}$ With QCD radiative corrections

${ }^{c}$ With constituent quark masses for the light quarks $m_{s}$ and $m_{\mathrm{d}}$

${ }^{d}$ With current quark masses for the light quarks $m_{s}$ and $m_{d}$

e With Coulomb plus linear potential model

${ }^{\mathrm{f}}$ With Coulomb plus harmonic oscillator potential model

${ }^{\mathrm{g}}$ With the Gaussian type wave functions

${ }^{\mathrm{h}}$ With the power-law type wave functions

${ }^{\mathrm{i}}$ With Coulomb plus linear potential model

${ }^{\mathrm{j}}$ With a dilation parameter $\kappa=0.54 \mathrm{GeV}$

${ }^{\mathrm{k}}$ With a dilation parameter $\kappa=0.68 \mathrm{GeV}$

${ }^{1}$ With Martin potential model [72]

${ }^{\mathrm{m}}$ With Cornell potential model [73]

${ }^{\mathrm{n}}$ With logarithmic potential model [74]

${ }^{\circ}$ With $m_{D^{*}} / f_{D^{*}}=8.6 \pm 0.3_{-0.9}^{+0.5}$ [76] and $m_{D^{*}}=2010.26(5) \mathrm{MeV}$ [1]

${ }^{\mathrm{p}}$ With harmonic plus Yukawa potential model [63]

The information about the decay constant $f_{D_{d}^{*}}$ has not yet been obtained experimentally by now. Some theoretical results on $f_{D_{d}^{*}}$ are listed in Table 3 . The theoretical discrepancies among various methods are obvious. In our calculation, as a conservative estimate, we will take the recent value $f_{D_{d}^{*}}=230 \pm 29 \mathrm{MeV}$ [28] from the light front quark model, which agrees basically with the values $f_{D_{d}^{*}}=234 \pm 6 \mathrm{MeV}$ [79] from the recent lattice QCD simulation.

After some simple computation with Eq. (8), we obtain the partial decay widths and branching ratios for the $D_{d}^{*-} \rightarrow$ $\ell^{-} \bar{v}_{\ell}$ decays as follows.

$\Gamma\left(D_{d}^{*-} \rightarrow \ell^{-} \bar{v}_{\ell}\right)=79_{-19}^{+22} \mu \mathrm{eV}, \quad$ for $\ell=e, \mu$,

$\Gamma\left(D_{d}^{*-} \rightarrow \tau^{-} \bar{v}_{\tau}\right)=5 \pm 1 \mu \mathrm{eV}$,

$\mathcal{B}\left(D_{d}^{*-} \rightarrow \ell^{-} \bar{v}_{\ell}\right)=\left(9.5_{-2.4}^{+2.9}\right) \times 10^{-10}, \quad$ for $\ell=e, \mu$,

$\mathcal{B}\left(D_{d}^{*-} \rightarrow \tau^{-} \bar{v}_{\tau}\right)=(0.6 \pm 0.2) \times 10^{-10}$.

These branching ratios are consistent with those of Ref. [89] if the different values of decay constants $f_{D_{d}^{*}}$ are considered. The relatively large uncertainties of branching ratios come from the uncertainties of mass $m_{D_{d}^{*}}$, width $\Gamma_{D_{d}^{*}}$, decay constant $f_{D_{d}^{*}}$ and the CKM element $\left|V_{c d}\right|$. To experimentally study the $D_{d}^{*-} \rightarrow \ell^{-} \bar{v}_{\ell}$ decays, more than $10^{11} D_{d}^{*}$ events are needed. Due to the short lifetime of lepton $\tau^{ \pm}$and the lepton number conservation in $\tau^{ \pm}$decays, additional neutri- nos will make the measurement of the $D_{d}^{*-} \rightarrow \tau^{-} \bar{\nu}_{\tau}$ decay to have a poor reconstruction efficiency and to be very challenging. Perhaps some $10^{12}$ or more $D_{d}^{*}$ events are necessarily required to study the $D_{d}^{*-} \rightarrow \tau^{-} \bar{\nu}_{\tau}$ decay.

Above the open charm production threshold, there are several charmonium resonances and charmonium-like structures decaying predominantly into pairs of charmed meson final states. The studies of Belle [90], BaBar [91] and CLEO-c $[92,93]$ collaborations have shown that there is a sharply peaked $D_{d}^{+} D_{d}^{*-}$ structure and a broad $D_{d}^{*+} D_{d}^{*-}$ plateau just above threshold, as illustrated in Fig. 4. Assuming the exclusive cross sections near threshold $\sigma\left(e^{+} e^{-} \rightarrow D_{d}^{+} D_{d}^{*-}\right) \sim 4 \mathrm{nb}$ and $\sigma\left(e^{+} e^{-} \rightarrow D_{d}^{*+} D_{d}^{*-}\right) \sim 3 \mathrm{nb}$, there will be about $10^{11}$ $D_{d}^{* \pm}$ events corresponding to the total integrated luminosity of $10 \mathrm{ab}^{-1}$ at future STCF, and about $5 \times 10^{11} D_{d}^{* \pm}$ events corresponding to a data sample of $50 \mathrm{ab}^{-1}$ at SuperKEKB. In addition, about $10^{12} \mathrm{Z}$ bosons will be produced on the on the schedule of the large international scientific project of Circular Electron Positron Collider (CEPC) [94] and $10^{13} \mathrm{Z}$ bosons at Future Circular $e^{+} e^{-}$Collider (FCC-ee) [95]. Considering the branching ratio $\mathcal{B}\left(Z \rightarrow D^{* \pm} X\right)=(11.4 \pm 1.3) \%$ [1], the $Z$ boson decays will yield more than $10^{11} D_{d}^{* \pm}$ events at the tera- $Z$ factories. So the $D_{d}^{*-} \rightarrow \ell^{-} \bar{v}_{\ell}$ decays could be investigated at Belle-II, SCTF, CEPC and FCC-ee experiments. 

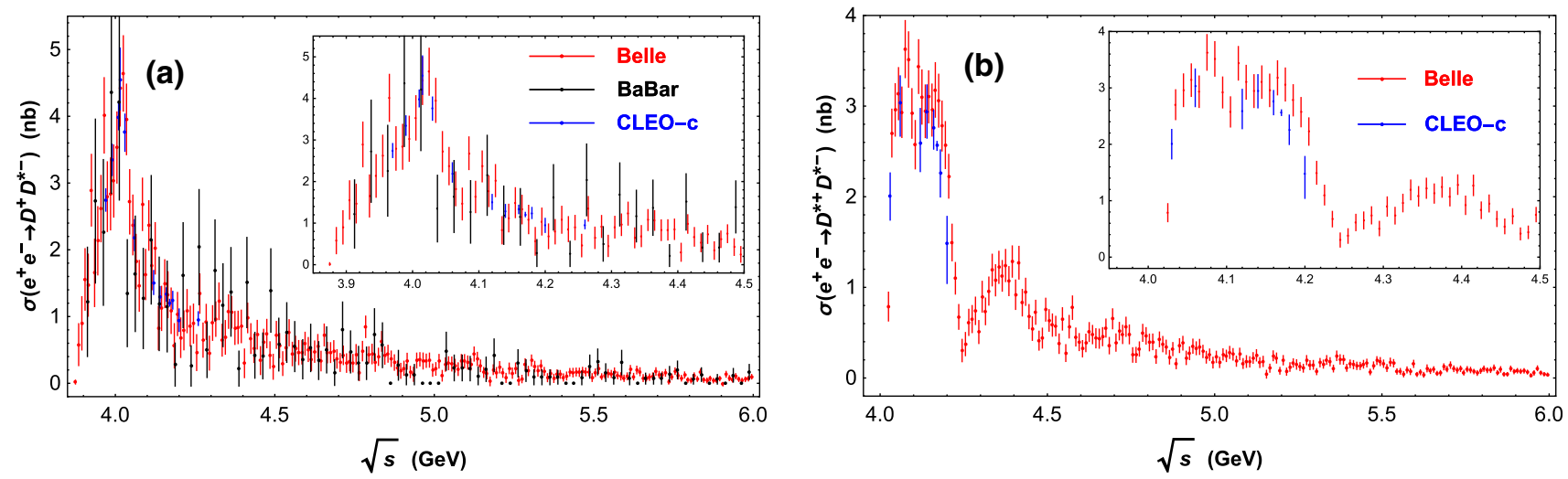

Fig. 4 The exclusive cross sections (in the unit of nb) as functions of $\sqrt{s}$ (in the unit of GeV) for $e^{+} e^{-} \rightarrow D^{+} D^{*-}$ in (a) and $e^{+} e^{-} \rightarrow D^{*+} D^{*-}$ in (b). The Belle, BaBar and CLEO-c data are from Refs. [90,91,93], respectively.

In hadron-hadron collisions, the inclusive cross sections for the $c \bar{c}$ pair and $D^{*+}$ meson production are measured to be $\sigma(p p \rightarrow c \bar{c} X)=2369 \pm 3 \pm 152 \pm 118 \mu \mathrm{b}$ and $\sigma\left(p p \rightarrow D^{*+} X\right)$ $=784 \pm 4 \pm 87 \pm 118 \mu \mathrm{b}$ at the center-of-mass energy of $\sqrt{\mathrm{s}}=$ $13 \mathrm{TeV}$ by the LHCb group, with the transverse momentum $p_{T}$ within the range of $1 \mathrm{GeV}<p_{T}<8 \mathrm{GeV}$ [55-57]. Some $2 \times 10^{14} D_{d}^{* \pm}$ events could be accumulated with the integrated luminosity $300 \mathrm{fb}^{-1}$ at LHCb. The total cross sections of charm and $D^{*+}$ production measured at $\sqrt{s}=7 \mathrm{TeV}$ by the ALICE group are $\sigma_{c \bar{c}}^{\text {tot }} \simeq 8.5 \mathrm{mb}$ and $\sigma_{D^{*+}}^{\text {tot }} \simeq 2.11 \mathrm{mb}$, respectively [96]. The total cross sections of charm production measured at $\sqrt{s}=7 \mathrm{TeV}$ by the ATLAS group are $\sigma_{c \bar{c}}^{\text {tot }} \simeq 8.6 \mathrm{mb}$ [97]. The $D^{*+}$ production cross section at ATLAS should be very close to that at ALICE based on an educated guess. In addition, the $D^{*}$ meson can also produced from $b$ decays with the fragmentation fraction about $f\left(b \rightarrow D_{d}^{*}\right) \simeq 23 \%$ [98]. The $b$-quark production cross sections at $\sqrt{s}=13 \mathrm{TeV}$ determined by LHCb and ALICE are about $\sigma(p p \rightarrow b \bar{b} X) \simeq$ $495 \mu \mathrm{b}[52,53]$ and $541 \mu \mathrm{b}$ [99], respectively. So more than $10^{13} D_{d}^{* \pm}$ events from $b$ decays could be accumulated with the integrated luminosity $300 \mathrm{fb}^{-1}$ at LHCb. All in all, the large cross section of $D^{*}$ meson plus the high luminosity at hadron-hadron collisions result in the abundant $D_{d}^{* \pm}$ events, and make the carefully experimental study of the $D_{d}^{*-} \rightarrow$ $e^{-} \bar{\nu}_{e}, \mu^{-} \bar{v}_{\mu}$ decays, even the $D_{d}^{*-} \rightarrow \tau^{-} \bar{\nu}_{\tau}$ decay, to be possible and practicable.

\section{$5 D_{s}^{*-} \rightarrow \ell^{-} \bar{v}_{\ell}$ decays}

The $D_{s}^{* \pm}$ mesons have explicitly nonzero quantum number of electric charges, charm and strange, $Q=C=S= \pm 1$. Considering the conservation of the charm and strange quantum number in the strong and electromagnetic interactions, and the mass of $D_{s}^{*}$ mesons, $m_{D_{s}^{*}}=2112.2(4) \mathrm{MeV}$ [1], being just above the threshold of $D_{s} \pi$ pair but below the threshold of $D K$ pair, the $D_{s}^{*} \rightarrow D_{s} \pi$ decays are the only allowable hadronic decay modes. However, the $D_{s}^{*} \rightarrow D_{s} \pi$ decays are highly suppressed due to four factors: (1) from the dynamical view, the $D_{s}^{*} \rightarrow D_{s} \pi$ decays are induced by the the electromagnetic interactions rather than the strong interactions because of the isospin non-conservation between the initial and final states, (2) from the perspective of the conservation of angular momentum, the orbital angular momentum of final states should be $L=1$, so the $D_{s}^{*} \rightarrow D_{s} \pi$ decays are induced by the contributions of the $P$-wave amplitudes, (3) from the phenomenological view, the $D_{s}^{*} \rightarrow D_{s} \pi$ decays are suppressed by the the Okubo-Zweig-Iizuka rules [3,100,101] because the quark lines of pion disconnect from those of the $D_{s}^{*} D_{s}$ system, (4) from the kinematic view, the phase spaces of final states are very compact because of $m_{D_{s}^{*}}-m_{D_{s}}-m_{\pi^{0}}$ $\sim 9 \mathrm{MeV}$. Hence, the branching ratio for the hadronic decay is very small $\mathcal{B}\left(D_{s}^{*} \rightarrow D_{s} \pi\right)=5.8(7) \%$ [1]. And the branching ratio of the electromagnetic radiative decay is dominant, $\mathcal{B}\left(D_{s}^{*} \rightarrow D_{s} \gamma\right)=93.5(7) \%$ [1]. Except for the $D_{s} \pi, D_{s} \gamma$ and $D_{s} e^{+} e^{-}$final states, other decay modes of the $D_{s}^{*}$ mesons have not yet been observed [1]. The $D_{s}^{*-} \rightarrow \ell^{-} \bar{v}_{\ell}$ weak decays are favored by the CKM element $\left|V_{c s}\right|$. The information about the $\left|V_{c S}\right| f_{D_{s}^{*}}$ can be experimentally obtained from the $D_{s}^{*-} \rightarrow \ell^{-} \bar{v}_{\ell}$ decays.

The direct determinations of the CKM element $\left|V_{c s}\right|$ come mainly from leptonic $D_{s}$ decays and semileptonic $D$ decays, as shown in Fig. 5. The uncertainties of $\left|V_{c s}\right|$ from the $D_{s}$ leptonic decays, about $1 \%$, are dominated by the experimental uncertainties. The uncertainties of $\left|V_{c s}\right|$ from the $D$ semileptonic decays, about $4 \%$, are dominated by the theoretical calculations of the form factors. It is wroth noting that the recent CKM element $\left|V_{c s}\right|$ determined by the BES-III group from the $D_{s}^{+} \rightarrow \mu^{+} v_{\mu}$ and $D_{s}^{+} \rightarrow \tau^{+} v_{\tau}$ decays based on available $6.32 \mathrm{fb}^{-1}$ data is $\left|V_{c s}\right|=0.978 \pm 0.009 \pm 0.014$ [102], where the systematic (second) uncertainties has outweighed the statistical (first) one. This value is very close to the precise result from the global fit, $\left|V_{c S}\right|=0.97320$ (11) [1] that will be used in this paper. 


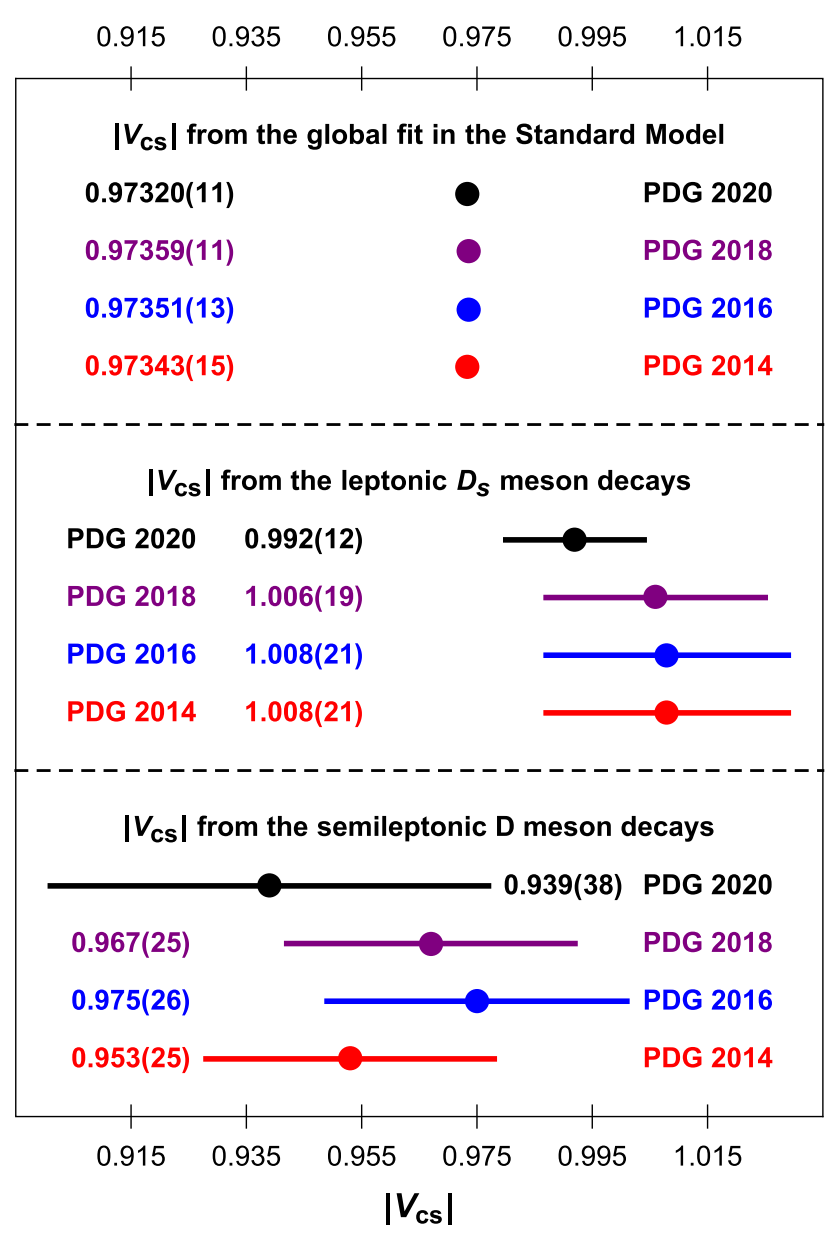

Fig. 5 The values of the CKM element $\left|V_{c s}\right|$ from PDG

By now, a relatively little information about the properties of the $D_{s}^{*}$ mesons is available. For example, the quantum number of $J^{P}$, the decay constant $f_{D_{s}^{*}}$, and the width $\Gamma_{D_{s}^{*}}$ have not yet been determined or confirmed explicitly by experiments. It is generally thought that the $J^{P}$ of the $D_{s}^{*}$ mesons is consistent with $1^{-}$from decay modes [105]. Some theoretical results on the decay constant $f_{D_{s}^{*}}$ are listed in Table 4. It can be seen that the theoretical results are various. The recent LQCD results on the decay constant from ETM [78], HPQCD [106] and $\chi$ QCD [79] groups are in reasonable agreement with each other within an error range. The latest decay constant $f_{D_{s}^{*}}=274 \pm 7 \mathrm{MeV}$ from LQCD calculation [79] will be used for an estimation for PLDCV of the $D_{s}^{*}$ mesons in this paper. The experimental upper limit of the decay width is $\Gamma_{D_{s}^{*}}<1.9 \mathrm{MeV}$ at the $90 \%$ confidence level set by the CLEO collaboration in 1995 [105]. An approximate relation for the decay width, $\Gamma_{D_{s}^{*}} \simeq \Gamma\left(D_{s}^{*} \rightarrow \gamma D_{s}\right)$, is often used in theoretical calculation. The radiative transition process, $D_{s}^{*} \rightarrow \gamma D_{s}$, is a parity conserving decay. The parity and angular momentum conservation implies that the orbital angular momentum of final states $L=1$. There are many theoretical calculation on the decay width $\Gamma_{D_{s}^{*}}$, for example,
Refs. [106-138]. The partial decay width for the magnetic dipole transition is generally written as [139],

$\Gamma(V \rightarrow P \gamma)=\frac{4}{3} \alpha_{\mathrm{em}} k_{\gamma}^{3} \mu_{V P}^{2}$,

with the definition of the magnetic dipole moment $\mu_{V P}$ and the momentum of photon $k_{\gamma}$ in the rest frame of the vector meson,

$\mu_{V P}=\left\langle P\left|\hat{\mu}_{z}\right| V\right\rangle=\left\langle P\left|\sum_{i} \frac{Q_{i}}{2 m_{i}} \hat{\sigma}_{z}\right| V\right\rangle$,

$k_{\gamma}=\frac{m_{V}^{2}-m_{P}^{2}}{2 m_{V}}$,

where $Q_{i}$ and $m_{i}$ are the electric charge in the unit of $|e|$ and mass of the constituent quark, respectively. With $m_{d} \approx 336$ $\mathrm{MeV}, m_{s} \approx 490 \mathrm{MeV}, m_{c} \approx 1500 \mathrm{MeV}$ and the

$\mu_{D_{d}^{*} D_{d}}=\frac{1}{6}\left(\frac{2}{m_{c}}-\frac{1}{m_{d}}\right)$,

$\mu_{D_{s}^{*} D_{s}}=\frac{1}{6}\left(\frac{2}{m_{c}}-\frac{1}{m_{s}}\right)$,

one can obtain $\Gamma\left(D_{d}^{*} \rightarrow \gamma D_{d}\right) \approx 1.8 \mathrm{keV}$ and $\Gamma\left(D_{s}^{*} \rightarrow \gamma D_{s}\right)$ $\approx 0.36 \mathrm{keV}$ [139]. The theoretical value of partial decay width $\Gamma\left(D_{d}^{*} \rightarrow \gamma D_{d}\right)$ is roughly consistent with the corresponding experimental data $\Gamma\left(D_{d}^{*} \rightarrow \gamma D_{d}\right)=\Gamma_{D_{d}^{*} \times \mathcal{B}}$ $\left(D_{d}^{*} \rightarrow \gamma D_{d}\right)=1.33 \pm 0.33 \mathrm{keV}$ within $2 \sigma$ regions [1]. For the moment, we will use $\Gamma_{D_{s}^{*}}=0.36 \mathrm{keV}$ in the calculation to give an estimate of branching ratios for the $D_{s}^{*-} \rightarrow \ell^{-} \bar{\nu}_{\ell}$ decays.

$\Gamma\left(D_{s}^{*-} \rightarrow \ell^{-} \bar{v}_{\ell}\right)=2.4 \pm 0.1 \mathrm{meV}, \quad$ for $\ell=e, \mu$,

$\Gamma\left(D_{s}^{*-} \rightarrow \tau^{-} \bar{\nu}_{\tau}\right)=0.28 \pm 0.02 \mathrm{meV}$,

$\mathcal{B}\left(D_{s}^{*-} \rightarrow \ell^{-} \bar{v}_{\ell}\right)=(6.7 \pm 0.4) \times 10^{-6}, \quad$ for $\ell=e, \mu$,

$\mathcal{B}\left(D_{s}^{*-} \rightarrow \tau^{-} \bar{v}_{\tau}\right)=(7.8 \pm 0.4) \times 10^{-7}$.

If considering the experimental measurement efficiency, there are at least more than $10^{7} D_{s}^{*}$ events to experimentally study the $D_{d}^{*-} \rightarrow \ell^{-} \bar{v}_{\ell}$ decays. And more than $10^{8} D_{s}^{*}$ events might be needed to explore the $D_{s}^{*-} \rightarrow \tau^{-} \bar{\nu}_{\tau}$ decay.

In the electron-positron collisions, the cross sections of $D_{s}^{+} D_{s}^{*-}$ and $D_{s}^{*+} D_{s}^{*-}$ production have been experimentally studied by the Belle [140], BaBar [141] and CLEO-c [92, 93] groups, as illustrated in Fig. 6. Assuming the exclusive cross sections near threshold $\sigma\left(e^{+} e^{-} \rightarrow D_{s}^{+} D_{s}^{*-}\right) \sim 1.0 \mathrm{nb}$ and $\sigma\left(e^{+} e^{-} \rightarrow D_{s}^{*+} D_{s}^{*-}\right) \sim 0.2 \mathrm{nb}$, there will be about $10^{10}$ $D_{s}^{* \pm}$ events corresponding to a data sample of $10 \mathrm{ab}^{-1}$ at STCF, and about $5 \times 10^{10} D_{s}^{* \pm}$ events corresponding to a data sample of $50 \mathrm{ab}^{-1}$ at SuperKEKB. In addition, considering the branching ratio $\mathcal{B}(Z \rightarrow c \bar{c})=(12.03 \pm 0.21) \%$ [1] and the fragmentation fraction $f\left(c \rightarrow D_{s}^{*}\right) \simeq 5.5 \%$ [142], there will be more than $6 \times 10^{9}\left(\right.$ and $\left.6 \times 10^{10}\right) D_{s}^{* \pm}$ events corresponding 
Table 4 The theoretical values of decay constant $f_{D_{s}^{*}}$ (in the unit of $\mathrm{MeV}$ ), where the legends including the footnotes are the same as those in 3

\begin{tabular}{|c|c|c|c|c|c|}
\hline NRQM & $326_{-17}^{+21}[61]$ & $344[62]^{\mathrm{a}}$ & $275[62]^{b}$ & $382.1[63]^{\mathrm{a}}$ & $303.5[63]^{\mathrm{b}}$ \\
\hline NRQM & $447[22]$ & $310[64]^{\mathrm{a}}$ & $212[64]^{b}$ & $384[65,66]$ & \\
\hline RQM & $315[22]$ & $335[65,66]$ & $362 \pm 15[67]^{\mathrm{c}}$ & $288 \pm 11[67]^{\mathrm{d}}$ & $272[103,104]$ \\
\hline LFQM & $290[68]^{\mathrm{e}}$ & $268[68]^{\mathrm{f}}$ & $338.7 \pm 29.7[69]^{\mathrm{g}}$ & $391.0 \pm 28.9[69]^{\mathrm{h}}$ & $314 \pm 6[27]$ \\
\hline LFQM & $260[24]^{\mathrm{i}}$ & $254.7_{-6.7}^{+6.3}[25]^{\mathrm{j}}$ & $289.7_{-4.5}^{+6.3}[25]^{\mathrm{k}}$ & $272_{-38}^{+39}[26]$ & $253 \pm 32[28]$ \\
\hline LFQM & $318.3_{-12.6}^{+15.3}[70]^{\mathrm{g}}$ & $330.9_{-9.0}^{+9.9}[70]^{\mathrm{h}}$ & $303[71]^{1}$ & $291[71]^{\mathrm{m}}$ & $302[71]^{\mathrm{n}}$ \\
\hline LQCD & $272 \pm 16_{-20}^{+3}[75]$ & $254 \pm 17[76]^{\circ}$ & $311 \pm 9[77]$ & & \\
\hline LQCD & $268.8 \pm 6.6[78]$ & $274 \pm 6[106]$ & $274 \pm 7$ [79] & & \\
\hline SR & $293_{-14}^{+19}[80-82]$ & $308 \pm 21[83]$ & $305.5 \pm 27.3[84]$ & $270 \pm 19[85]$ & \\
\hline Other & $240[71]^{\mathrm{p}}$ & $307 \pm 18[86]$ & $375 \pm 24[87]$ & $242[88]$ & \\
\hline
\end{tabular}

${ }^{a}$ Without QCD radiative corrections

${ }^{\mathrm{b}}$ With QCD radiative corrections

${ }^{c}$ With constituent quark masses for the light quarks $m_{s}$ and $m_{\mathrm{d}}$

${ }^{\mathrm{d}}$ With current quark masses for the light quarks $m_{s}$ and $m_{d}$

${ }^{\mathrm{e}}$ With Coulomb plus linear potential model

${ }^{\mathrm{f}}$ With Coulomb plus harmonic oscillator potential model

${ }^{g}$ With the Gaussian type wave functions

${ }^{\mathrm{h}}$ With the power-law type wave functions

${ }^{\mathrm{i}}$ With Coulomb plus linear potential model

${ }^{\mathrm{j}}$ With a dilation parameter $\kappa=0.54 \mathrm{GeV}$

${ }^{\mathrm{k}}$ With a dilation parameter $\kappa=0.68 \mathrm{GeV}$

${ }^{l}$ With Martin potential model [72]

${ }^{\mathrm{m}}$ With Cornell potential model [73]

${ }^{\mathrm{n}}$ With logarithmic potential model [74]

${ }^{\circ}$ With $m_{D_{s}^{*}} / f_{D_{s}^{*}}=8.3 \pm 0.2 \pm 0.5[76]$ and $m_{D_{s}^{*}}=2112.2(4) \mathrm{MeV}[1]$

${ }^{\mathrm{p} W i t h}$ harmonic plus Yukawa potential model [63]
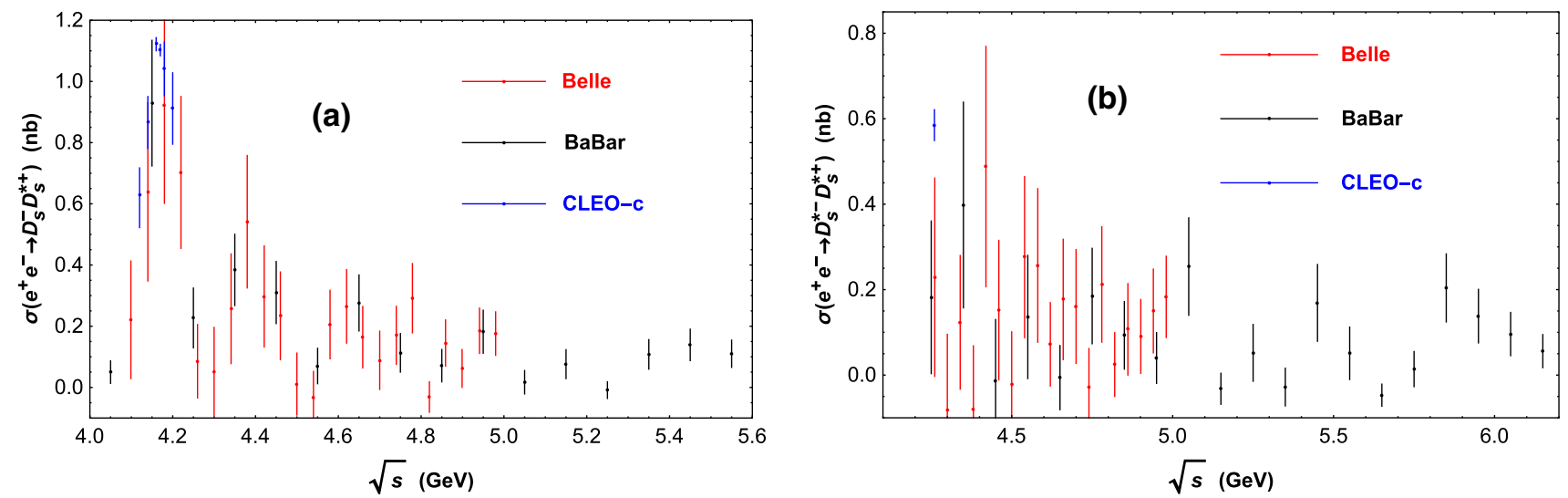

Fig. 6 The exclusive cross sections (in the unit of nb) as functions of $\sqrt{s}$ (in the unit of GeV) for $e^{+} e^{-} \rightarrow D_{s}^{-} D_{s}^{*+}$ in (a) and $e^{+} e^{-} \rightarrow D_{s}^{*-} D_{s}^{*+}$ in (b). The Belle, BaBar and CLEO-c data are from Ref. [93,140,141], respectively

to $10^{12}$ [94] (and $10^{13}$ [95]) $Z$ bosons at the future CEPC (and FCC-ee). So the $D_{s}^{*-} \rightarrow \ell^{-} \bar{v}_{\ell}$ decays (with $\ell=e, \mu$ and $\tau$ ) could be measured at Belle-II, SCTF, CEPC and FCC-ee experiments.

In hadron-hadron collisions, the inclusive cross sections for the $c \bar{c}$ pair production are $\sigma(p p \rightarrow c \bar{c} X) \simeq 2.4 \mathrm{mb}$ at the center-of-mass energy of $\sqrt{s}=13 \mathrm{TeV}$ at LHCb [55-57], $\sigma_{c \bar{c}}^{\text {tot }} \simeq 8.5 \mathrm{mb}$ and $8.6 \mathrm{mb}$ at $\sqrt{s}=7 \mathrm{TeV}$ at ALICE [96] and
ATLAS [97], respectively. With the fragmentation fraction $f\left(c \rightarrow D_{s}^{*}\right) \simeq 5.5 \%$ [142], there will be about $4 \times 10^{13} D_{s}^{* \pm}$ events corresponding a data sample of $300 \mathrm{fb}^{-1}$ at LHCb, and more $D_{s}^{* \pm}$ events available at ALICE and ATLAS. So the $D_{s}^{*-} \rightarrow e^{-} \bar{v}_{e}, \mu^{-} \bar{v}_{\mu}, \tau^{-} \bar{v}_{\tau}$ decays could be measured precisely at LHCb, ALICE and ATLAS experiments. 


\section{$6 B_{u}^{*-} \rightarrow \ell^{-} \bar{v}_{\ell}$ decays}

The experimental information about the $B_{u}^{*}$ mesons are very scarce. The already known information about the $B_{u}^{*}$ mesons are their quark composition $b \bar{u}$ with the quark model assignment, the isospin $I=1 / 2$, the spin-parity quantum number $J^{P}=1^{-}$and the mass $m_{B_{u}^{*}}=5324.70(21) \mathrm{MeV}$ [1]. Due to the mass difference $m_{B_{u}^{*}}-m_{B_{u}}=45 \mathrm{MeV}<m_{\pi}$, the electromagnetic radiative transition $B_{u}^{*} \rightarrow B_{u} \gamma$ certainly will be the important and dominant decay mode. The photon in the $B_{u}^{*} \rightarrow B_{u} \gamma$ decay is very soft, with the momentum $k_{\gamma} \sim$ $45 \mathrm{MeV}$ in the center-of-mass of the $B_{u}^{*}$ mesons. No signal event of the $B_{u}^{*} \rightarrow B_{u} \gamma$ decay has yet been found. The $B_{u}^{*-} \rightarrow \ell^{-} \bar{\nu}_{\ell}$ decays offer a complementary decay modes of the $B_{u}^{*}$ meson. It can be seen from Eq. (8) that the information about $\left|V_{u b}\right| f_{B_{u}^{*}}$ could be obtained, however, the partial width for the purely leptonic decays $B_{u}^{*-} \rightarrow \ell^{-} \bar{\nu}_{\ell}$ are highly suppressed by the CKM element of $\left|V_{u b}\right|^{2} \sim \mathcal{O}\left(\lambda^{6}\right)$.

The precise determinations of the CKM element $V_{u b}=$ $\left|V_{u b}\right| e^{-i \gamma}$ are very central and important to verify the CKM picture of SM, where $\gamma$ is the angle of the unitarity triangle of $V_{u d} V_{u b}^{*}+V_{c d} V_{c b}^{*}+V_{t d} V_{t b}^{*}=0$. The experimental determination of $\left|V_{u b}\right|$ from the inclusive $B \rightarrow X_{u} \ell \bar{v}_{\ell}$ decay is complicated mainly by the large backgrounds from the CKM-favored $B \rightarrow X_{c} \ell \bar{v}_{\ell}$ decay. The experimental extraction of $\left|V_{u b}\right|$ from exclusive $B \rightarrow \pi \ell \bar{v}_{\ell}$ decay is subject to the form factors calculated with the lattice QCD or QCD sum rules. The latest values obtained from inclusive and exclusive determinations are [1]

$$
\begin{aligned}
& \left|V_{u b}\right| \times 10^{3}=4.25 \pm 0.12_{\exp }^{+0.15}{ }_{-0.14}^{+0 o} \pm 0.23_{\triangle B F} \\
& \quad=4.25_{-0.29}^{+0.30} \quad \text { (inclusive) } \\
& \left|V_{u b}\right| \times 10^{3}=3.70 \pm 0.10_{\exp } \pm 0.12_{\text {theo }} \\
& \quad=3.70 \pm 0.16 \quad \text { (exclusive) }
\end{aligned}
$$

It is clearly seen that (1) the difference between inclusive and exclusive determinations of $\left|V_{u b}\right|$ is obvious. (2) The best determinations of $\left|V_{u b}\right|$ are from exclusive semileptonic decays, with a precision of about $4 \%$. The experimental errors for the exclusive semileptonic decays are expected to decrease from the current $2.7 \%$ to $1.2 \%$ based on a dataset of $50 \mathrm{ab}^{-1}$ at Belle-II experiments [43,44]. (3) The theoretical uncertainties are larger than experimental ones. Moreover, $\left|V_{u b}\right|$ can also be experimentally determined from the leptonic decay $B_{u} \rightarrow \tau \bar{\nu}_{\tau}$ and the semileptonic hyperon decay $\Lambda_{b} \rightarrow p \mu \bar{v}_{\mu}$. The constraint from the global fit gives $\left|V_{u b}\right|=$ $\left(3.61_{-0.09}^{+0.11}\right) \times 10^{-3}$ [1], which will be used in our calculation.

Some theoretical results on the decay constant $f_{B_{u}^{*}}$ are collected in Table 5. The recent result $f_{B_{u}^{*}}=185.9 \pm 7.2 \mathrm{MeV}$ from LQCD calculation [78] will be used in this paper. There are many theoretical calculation on the decay width $\Gamma_{B_{u}^{*}}$, for example, Refs. [119-138]. With the formula of Eq. (32), the quark mass $m_{u}=336 \mathrm{MeV}$ [139] and $m_{b}=4.78 \mathrm{GeV}$ [1], and the magnetic dipole momentum

$\mu_{B_{u}^{*} B_{u}}=\frac{1}{6}\left(\frac{2}{m_{u}}-\frac{1}{m_{b}}\right)$,

one can obtain $\Gamma_{B_{u}^{*}} \simeq \Gamma\left(B_{u}^{*} \rightarrow \gamma B_{u}\right) \simeq 820 \mathrm{eV}$. The partial decay width and branching ratios for the $B_{u}^{*-} \rightarrow \ell^{-} \bar{v}_{\ell}$ decays are

$$
\begin{aligned}
\Gamma\left(B_{u}^{*-} \rightarrow \ell^{-} \bar{v}_{\ell}\right) & =0.25_{-0.03}^{+0.04} \mu \mathrm{eV}, \quad \text { for } \ell=e, \mu, \\
\Gamma\left(B_{u}^{*-} \rightarrow \tau^{-} \bar{v}_{\tau}\right) & =0.20_{-0.02}^{+0.03} \mu \mathrm{eV}, \\
\mathcal{B}\left(B_{u}^{*-} \rightarrow \ell^{-} \bar{v}_{\ell}\right) & =(3.0 \pm 0.4) \times 10^{-10}, \quad \text { for } \ell=e, \mu, \\
\mathcal{B}\left(B_{u}^{*-} \rightarrow \tau^{-} \bar{v}_{\tau}\right) & =(2.5 \pm 0.4) \times 10^{-10} .
\end{aligned}
$$

It is expected that there should be at least more than $10^{11}$ $B_{u}^{*}$ events available for experimental study of the $B_{u}^{*-} \rightarrow$ $\ell^{-} \bar{v}_{\ell}$ decays.

The experimental study has shown that the exclusive cross sections for the final states of $B \bar{B}^{*}, B^{*} \bar{B}^{*}$ and $B \bar{B}^{*} \pi$ will have a large share of the total $b \bar{b}$ cross sections above the open bottom threshold, for example [1],

$$
\begin{aligned}
& \mathcal{B}\left(Z_{b}(10610) \rightarrow B^{+} \bar{B}^{* 0}+B^{*+} \bar{B}^{0}\right)=85.6_{-2.9}^{+2.1} \%, \\
& \mathcal{B}\left(Z_{b}(10650) \rightarrow B^{*+} \bar{B}^{* 0}\right)=74_{-6}^{+4} \%, \\
& \mathcal{B}\left(\Upsilon(5 S) \rightarrow B \bar{B}^{*}+\text { c.c. }\right)=13.7 \pm 1.6 \% \\
& \mathcal{B}\left(\Upsilon(5 S) \rightarrow B^{*} \bar{B}^{*}\right)=38.1 \pm 3.4 \% \\
& \mathcal{B}\left(\Upsilon(5 S) \rightarrow B \bar{B}^{*} \pi+B^{*} \bar{B} \pi\right)=7.3 \pm 2.3 \%, \\
& \mathcal{B}\left(\Upsilon(5 S) \rightarrow B^{*} \bar{B}^{*} \pi\right)=1.0 \pm 1.4 \% .
\end{aligned}
$$

There are about $36 \times 10^{6} \Upsilon(5 S)$ events corresponding to the dataset of $121 \mathrm{fb}^{-1}$ at Belle experiments at the disposal $[43,44]$. About $1.5 \times 10^{10} \Upsilon(5 S)$ events with a dataset 50 $\mathrm{ab}^{-1}$ at Belle-II are an outside estimate. Assuming the inclusive branching ratio $\mathcal{B}\left(\Upsilon(5 S) \rightarrow B_{u}^{*} X\right) \simeq 30 \%$, there will be some $4.5 \times 10^{9} B_{u}^{*}$ events at most at Belle-II. And it is more important that the vast majority of the data will be taken at $\Upsilon(4 S)$ resonance rather than $\Upsilon(5 S)$ mesons at Belle-II experiments, and $\Upsilon(4 S)$ lies below the $B \bar{B}^{*}$ threshold. So the probability of direct observation of the $B_{u}^{*-} \rightarrow \ell^{-} \bar{\nu}_{\ell}$ decays at Belle-II experiments should be very tiny. Considering about $10^{13} Z$ bosons at FCC-ee [95] and branching ratio $\mathcal{B}(Z \rightarrow b \bar{b})$ $=12.03 \pm 0.21 \%$ [1] , and assuming the fragmentation fraction $f\left(b \rightarrow B_{u}^{*}\right) \sim 20 \%$ [146], there will be more than $4 \times 10^{11}$ $B_{u}^{*}$ events to search for the $B_{u}^{*-} \rightarrow \ell^{-} \bar{v}_{\ell}$ decays. The $b$-quark production cross sections at the center-of-mass energy $\sqrt{s}=$ $13 \mathrm{TeV}$ is about $\sigma(p p \rightarrow b \bar{b} X) \simeq 495 \mu \mathrm{b}$ at LHCb $[52,53]$. There will be more than $5 \times 10^{13} B_{u}^{*}$ events with a dateset of $300 \mathrm{fb}^{-1}$ at LHCb and fragmentation fraction $f\left(b \rightarrow B_{u}^{*}\right) \sim$ $20 \%$. Hence, the $B_{u}^{*-} \rightarrow e^{-} \bar{v}_{e}, \mu^{-} \bar{v}_{\mu}, \tau^{-} \bar{v}_{\tau}$ decays could be investigated at FCC-ee and LHCb experiments in the future. 
Table 5 The theoretical values of decay constant $f_{B_{u}^{*}}$ (in the unit of $\mathrm{MeV}$ ), where the legends are the same as those in Table 3

\begin{tabular}{|c|c|c|c|c|c|}
\hline NRQM & $280[22]$ & $151_{-13}^{+15}[61]$ & $234.7[63]^{\mathrm{a}}$ & $225.1[63]^{\mathrm{b}}$ & \\
\hline NRQM & $196[64]^{\mathrm{a}}$ & $182[64]^{\mathrm{b}}$ & $213[65,66]$ & $242.37[143]^{\mathrm{a}}$ & $232.47[143]^{\mathrm{b}}$ \\
\hline RQM & 219 [22] & $195[65,66]$ & $252 \pm 10[67]^{c}$ & $193 \pm 8[67]^{\mathrm{d}}$ & \\
\hline LFQM & $188[24]^{\mathrm{e}}$ & $198.7_{-11.3}^{+4.9}[25]^{\mathrm{f}}$ & $193.1_{-4.6}^{+4.3}[25]^{\mathrm{g}}$ & $196_{-27}^{+28}[26]$ & $172_{-23}^{+24} \pm 6[28]$ \\
\hline LFQM & $204[68]^{\mathrm{h}}$ & $193[68]^{\mathrm{i}}$ & $225 \pm 38[69]^{j}$ & $249_{-42}^{+44}[69]^{\mathrm{k}}$ & $205 \pm 5[27]$ \\
\hline LFQM & $201.9_{-41.4}^{+43.2}[70]^{\mathrm{j}}$ & $220.2_{-46.2}^{+49.1}[70]^{\mathrm{k}}$ & $227[71]^{1}$ & $211[71]^{\mathrm{m}}$ & $228[71]^{\mathrm{n}}$ \\
\hline LQCD & $196 \pm 24_{-2}^{+39}[75]$ & $190 \pm 28[76]^{\circ}$ & $185.9 \pm 7.2[78]$ & $175 \pm 6[144]$ & \\
\hline $\mathrm{SR}$ & $210_{-12}^{+10}[80-82]$ & $213 \pm 18[83]$ & $209 \pm 8[85]$ & $209_{-22}^{+23}[138]$ & $181.8 \pm 13.7[145]$ \\
\hline Other & $138[71]^{\mathrm{p}}$ & $200 \pm 10[86]$ & $238 \pm 18[87]$ & $164[88]$ & \\
\hline
\end{tabular}

\footnotetext{
${ }^{a}$ Without QCD radiative corrections

${ }^{\mathrm{b}}$ With QCD radiative corrections

${ }^{\mathrm{c}}$ With $f_{B_{u}^{*}}=f_{B_{d}^{*}}$, and the constituent quark masses for the light quarks $s$ and $d$

${ }^{\mathrm{d}}$ With $f_{B_{u}^{*}}=f_{B_{d}^{*}}$, and the current quark masses for the light quarks $s$ and $d$

${ }^{\mathrm{e}}$ With Coulomb plus linear potential model

${ }^{\mathrm{f}}$ With a dilation parameter $\kappa=0.54 \mathrm{GeV}$

gith a dilation parameter $\kappa=0.68 \mathrm{GeV}$

${ }^{\mathrm{h}}$ With Coulomb plus linear potential model

${ }^{\mathrm{i}}$ With Coulomb plus harmonic oscillator potential model

${ }^{\mathrm{j}}$ With the Gaussian type wave functions

${ }^{\mathrm{k}}$ With the power-law type wave functions

${ }^{l}$ With Martin potential model [72]

${ }^{\mathrm{m}}$ With Cornell potential model [73]

${ }^{\mathrm{n}}$ With logarithmic potential model [74]

${ }^{\circ}$ With $m_{B^{*}} / f_{B^{*}}=28 \pm 1_{-4}^{+3}[76]$ and $m_{B^{*}}=5324.70(21) \mathrm{MeV}$ [1]

pWith harmonic plus Yukawa potential model [63]
}

\section{$7 B_{c}^{*-} \rightarrow \ell^{-} \bar{v}_{\ell}$ decays}

According to the conventional quark-model assignments, the $B_{c}^{*}$ mesons consist of two heavy quarks with different flavor numbers $B=C=-Q= \pm 1$. Up to today, the experimental information of the $B_{c}^{*}$ meson is still very limited. For example, the potential candidate of the $B_{c}^{*}$ meson has not yet been determined. It is generally believed that the mass of the $B_{c}^{*}$ meson should be in the region between $m_{B_{c}}$ $=6274.47 \pm 0.27 \pm 0.17 \mathrm{MeV}$ recently measured by $\mathrm{LHCb}$ [147] and $m_{B_{c}(2 S)}=6872.1 \pm 1.3 \pm 0.1 \pm 0.8 \mathrm{MeV}$ obtained by $\mathrm{LHCb}$ [148] (or $6871.0 \pm 1.2 \pm 0.8 \pm 0.8 \mathrm{MeV}$ given by CMS [149]), where the $B_{c}, B_{c}^{*}$ and $B_{c}(2 S)$ particles correspond to the sibling isoscalar states with quantum numbers of $n^{2 S+1} L_{J}=1{ }^{1} S_{0}, 1^{3} S_{1}$ and $2^{1} S_{0}$, respectively. So the branching ratios for the strong decays $B_{c}^{*} \rightarrow B D$ are zero, because $B_{c}^{*}$ meson is below the $B D$ pair threshold. The experimental particle physicists are earnestly looking for and identifying the $B_{c}^{*}$ meson, a long-expected charming beauty. For the moment, almost all of the information available about the properties of $B_{c}^{*}$ meson (such as the mass, decay constant, lifetime, decay modes and so on) come from theoretical estimates. There are too many estimations on the $B_{c}^{*}$ meson mass with various theoretical models, for example, in Refs. [150-207]. The recent result from lattice QCD calculation, $m_{B_{c}^{*}}=6331 \pm 7 \mathrm{MeV}$ [192], which are basically consistent with other estimations, will be used in this paper. Clearly, it is foreseeable that the isospin violating decay $B_{c}^{*}$ $\rightarrow B_{c} \pi$ is explicitly forbidden by the law of energy conservation, because of $m_{B_{c}^{*}}-m_{B_{c}} \simeq 57 \mathrm{MeV}<m_{\pi}$. Hence, the electromagnetic radiative transition $B_{c}^{*} \rightarrow B_{c} \gamma$ should be the dominant decay mode. In addition, the photon in the magnetic dipole transition $B_{c}^{*} \rightarrow B_{c} \gamma$ is very soft in the rest frame of the $B_{c}^{*}$ meson. This might be one main reason why the unambiguously experimental identification of the $B_{c}^{*}$ meson is very challenging. As an important complementary decay modes, the $B_{c}^{*}$ meson has very rich weak decay channels, which could be approximately classified into three classes: (1) the valence $b$ quark weak decay accompanied by the spectator $c$ quark, (2) the valence $c$ quark weak decay accompanied by the spectator $b$ quark, and (3) the $b$ and $c$ quarks annihilation into a virtual $W$ boson. The purely leptonic decays $B_{c}^{*-} \rightarrow \ell^{-} \bar{v}_{\ell}$ belong to the third case, which are favored by the CKM element $\left|V_{c b}\right|$. And the information of $\left|V_{c b}\right| f_{B_{c}^{*}}$ could be obtained from the $B_{c}^{*-} \rightarrow \ell^{-} \bar{v}_{\ell}$ decays.

The current values of the CKM element $\left|V_{c b}\right|$ come mainly from inclusive and exclusive semileptonic decays of $B$ meson to charm [1]. The average values obtained from inclusive $b \rightarrow c \ell \bar{v}_{\ell}$ decays and exclusive $B \rightarrow D^{(*)} \ell \bar{v}_{\ell}$ decays are $\left|V_{c d}\right| \times 10^{3}=42.2(8)$ and 39.5(9), respectively [1]. The lepton flavor non-universality in the ratio $R\left(D^{(*)}\right)$ complicate the determination of $\left|V_{c b}\right|$. In addition, $\left|V_{c b}\right|$ can also be 
Table 6 The theoretical values of decay constant $f_{B_{c}^{*}}$ (in the unit of $\mathrm{MeV}$ ), where the legends are the same as those in Table 3

\begin{tabular}{|c|c|c|c|c|c|}
\hline NRQM & $562[65,66]$ & 434.64 [193] & $544.3[204]$ & & \\
\hline RQM & $503[65,66]$ & $510 \pm 80[164]^{\mathrm{a}}$ & $456 \pm 70[164]^{\mathrm{b}}$ & $460 \pm 60[164]^{c}$ & \\
\hline LFQM & $391_{-5}^{+4}[24]$ & $440_{-52}^{+51}[26]$ & $387[69]^{\mathrm{d}}$ & $423[69]^{\mathrm{e}}$ & $465 \pm 7[27]$ \\
\hline LFQM & $473.4 \pm 18.2[70]^{\mathrm{d}}$ & $487.6 \pm 19.2[70]^{\mathrm{e}}$ & $398[208]^{\mathrm{f}}$ & $551[208]^{\mathrm{g}}$ & $474 \pm 42$ [209] \\
\hline LQCD & $422 \pm 13[144]$ & $387 \pm 12[210]$ & & & \\
\hline SR & $384 \pm 32[188]^{\mathrm{h}}$ & $415 \pm 31[188]^{\mathrm{i}}$ & $300 \pm 30$ [199] & $442 \pm 44[201]^{\mathrm{j}}$ & $387 \pm 15[201]^{\mathrm{k}}$ \\
\hline Other & $453 \pm 20[86]$ & $418 \pm 24[87]$ & $471[194]$ & & \\
\hline
\end{tabular}

${ }^{a}$ With Martin potential model [72]

${ }^{\mathrm{b}}$ With Coulomb plus linear potential model

${ }^{c}$ Obtained from the scaling relation

${ }^{\mathrm{d}}$ With the Gaussian type wave functions

${ }^{\mathrm{e}}$ With the power-law type wave functions

${ }^{\mathrm{f}}$ With Coulomb plus linear potential model

gWith Coulomb plus harmonic oscillator potential model

${ }^{\mathrm{h}}$ With the current quark mass

${ }^{\mathrm{i}}$ With the pole quark mass

${ }^{j}$ With inputs from the inverse Laplace-type model

${ }^{\mathrm{k}}$ With inputs from Heavy Quark Symmetry

obtained from the PLDCM $B_{c}^{-} \rightarrow \ell \bar{v}_{\ell}$ decays, although none of the measurements has reached a competitive level of precision due to either the serious helicity suppression for $B_{c}^{-} \rightarrow$ $e \bar{v}_{e}, \mu \bar{v}_{\mu}$ decays or other additional neutrinos from $\tau$ decay for $B_{c}^{-} \rightarrow \tau \bar{v}_{\tau}$ decay. The global SM fit value is $\left|V_{c b}\right|=$ $40.53_{-0.61}^{+0.83} \times 10^{-3}$ [1], which will be used in this paper.

Both valence quarks of the $B_{c}^{(*)}$ mesons are regarded as heavy quarks. Their Compton wave lengths $\sim 1 / m_{b, c}$ are much shorter than a typical hadron size. The spin-flavor symmetry in the heavy quark limit would lead to an approximation between decay constants $f_{B_{c}} \approx f_{B_{c}^{*}}$. Some theoretical results on the decay constant $f_{B_{c}^{*}}$ are collected in Table 6. The recent lattice QCD calculation $f_{B_{c}^{*}}=387 \pm 12 \mathrm{MeV}$ [210] will be used in this paper. As it is well known that the magnetic momentum of both $b$ and $c$ quarks are inversely proportional to their mass. The magnetic dipole momentum

$\mu_{B_{c}^{*} B_{c}}=\frac{1}{6}\left(\frac{2}{m_{c}}-\frac{1}{m_{b}}\right)$,

should be very small. With the quark mass $m_{c}=1.5 \mathrm{GeV}$ and $m_{b}=4.78 \mathrm{GeV}$, one can obtain $\Gamma_{B_{c}^{*}} \simeq \Gamma\left(B_{c}^{*} \rightarrow \gamma B_{c}\right) \simeq$ $60 \mathrm{eV}$ using the formula of Eq. (32).

The partial decay width and branching ratios for the $B_{c}^{*-}$ $\rightarrow \ell^{-} \bar{v}_{\ell}$ decays are estimated to be,

$$
\begin{aligned}
\Gamma\left(B_{c}^{*-} \rightarrow \ell^{-} \bar{v}_{\ell}\right) & =225_{-21}^{+25} \mu \mathrm{eV}, \quad \text { for } \ell=e, \mu, \\
\Gamma\left(B_{c}^{*-} \rightarrow \tau^{-} \bar{v}_{\tau}\right) & =198_{-18}^{+22} \mu \mathrm{eV}, \\
\mathcal{B}\left(B_{c}^{*-} \rightarrow \ell^{-} \bar{v}_{\ell}\right) & =\left(3.8_{-0.3}^{+0.4}\right) \times 10^{-6}, \quad \text { for } \ell=e, \mu, \\
\mathcal{B}\left(B_{c}^{*-} \rightarrow \tau^{-} \bar{v}_{\tau}\right) & =\left(3.3_{-0.3}^{+0.4}\right) \times 10^{-6} .
\end{aligned}
$$

To experimentally investigate the $B_{c}^{*-} \rightarrow \ell^{-} \bar{\nu}_{\ell}$ decays, there should be at least more than $10^{7} B_{c}^{*}$ events available.
More than $10^{12} Z$ bosons are expected at the future $e^{+} e^{-}$ colliders of CEPC [94] and FCC-ee [95]. Considering the branching ratio $\mathcal{B}(Z \rightarrow b \bar{b})=12.03 \pm 0.21 \%$ [1] and fragmentation fraction $f\left(b \rightarrow B_{c}^{*}\right) \sim 6 \times 10^{-4}$ [211-213], there will be more than $10^{8} B_{c}^{*}$ events to search for the $B_{c}^{*-} \rightarrow$ $e^{-} \bar{v}_{e}, \mu^{-} \bar{v}_{\mu}, \tau^{-} \bar{v}_{\tau}$ decays. In addition, the $B_{c}^{*}$ production cross sections at LHC are estimated to be about $100 \mathrm{nb}$ for $p p$ collisions at $\sqrt{s}=13 \mathrm{TeV}$, about $8 \mathrm{mb}$ for $p$ - $\mathrm{Pb}$ collisions at $\sqrt{s}=8.16 \mathrm{TeV}$ and some $920 \mathrm{mb}$ for $\mathrm{Pb}-\mathrm{Pb}$ collisions at $\sqrt{s}=5.02 \mathrm{TeV}$, respectively [214]. There will be more than $3 \times 10^{10} B_{c}^{*}$ events corresponding to a dataset of $300 \mathrm{fb}^{-1}$ at LHCb for $p p$ collisions. Hence, the $B_{c}^{*-} \rightarrow e^{-} \bar{v}_{e}, \mu^{-} \bar{v}_{\mu}$, $\tau^{-} \bar{\nu}_{\tau}$ decays are expected to be carefully measured at $\mathrm{LHCb}$ experiments in the future.

\section{Summary}

The mass of the charged vector mesons are generally larger than that of the corresponding ground pseudoscalar mesons. The vector mesons decay mainly through the strong or/and electromagnetic interactions. These facts will inevitably result in that the branching ratios of the vector meson weak decays are often very tiny. Inspired by the potential prospects of existing and coming high-luminosity experiments, more and more experimental data will be accumulated, and higher measurement precision level will be reached. The probabilities of experimental investigation on the purely leptonic decays of charged vector mesons are discussed in this paper. We found that (1) for both $\rho^{ \pm}$and $K^{* \pm}$ mesons, their widths are large due to the dominance of strong decay. Their PLDCV branching ratios are estimated at the order of $\mathcal{O}\left(10^{-13}\right)$. Although extremely complicated and difficult, the PLDCV 
Table 7 The probabilities of experimental investigation on PLDCV, where $\mathcal{B}$ denotes the branching ratio, and the symbol $\star$ denotes that the PLDCV process might be experimentally accessible in the future

\begin{tabular}{|c|c|c|c|c|c|c|}
\hline Decay modes & $\mathcal{B}$ & Belle-II & SCTF/STCF & CEPC & FCC-ee & $\mathrm{LHCb}$ \\
\hline$\rho^{-} \rightarrow e^{-} \bar{v}_{e}, \mu^{-} \bar{v}_{\mu}$ & $\mathcal{O}\left(10^{-13}\right)$ & & & & & $\star$ \\
\hline$K^{*-} \rightarrow e^{-} \bar{v}_{e}, \mu^{-} \bar{v}_{\mu}$ & $\mathcal{O}\left(10^{-13}\right)$ & & & & & $\star$ \\
\hline$D_{d}^{*-} \rightarrow e^{-} \bar{v}_{e}, \mu^{-} \bar{v}_{\mu}, \tau^{-} \bar{v}_{\tau}$ & $\mathcal{O}\left(10^{-10}\right)$ & $\star$ & $\star$ & $\star$ & $\star$ & $\star$ \\
\hline$D_{s}^{*-} \rightarrow e^{-} \bar{v}_{e}, \mu^{-} \bar{v}_{\mu}, \tau^{-} \bar{v}_{\tau}$ & $\mathcal{O}\left(10^{-6}\right)$ & $\star$ & $\star$ & $\star$ & $\star$ & $\star$ \\
\hline$B_{u}^{*-} \rightarrow e^{-} \bar{v}_{e}, \mu^{-} \bar{v}_{\mu}, \tau^{-} \bar{v}_{\tau}$ & $\mathcal{O}\left(10^{-10}\right)$ & & & 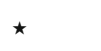 & $\star$ & $\star$ \\
\hline$B_{c}^{*-} \rightarrow e^{-} \bar{v}_{e}, \mu^{-} \bar{v}_{\mu}, \tau^{-} \bar{v}_{\tau}$ & $\mathcal{O}\left(10^{-6}\right)$ & & & $\star$ & $\star$ & $\star$ \\
\hline
\end{tabular}

decays $\rho^{ \pm}, K^{* \pm} \rightarrow e^{-} \bar{v}_{e}, \mu^{-} \bar{v}_{\mu}$ might be measurable due to the huge data of the $\rho^{ \pm}$and $K^{* \pm}$ mesons at LHCb. (2) The PLDCV $D_{S}^{*}$ decays are favored by the CKM element $\left|V_{c s}\right|$. Their branching ratios are about $\mathcal{O}\left(10^{-6}\right)$. The PLDCV decays $D_{d, s}^{*} \rightarrow e^{-} \bar{v}_{e}, \mu^{-} \bar{v}_{\mu}, \tau^{-} \bar{v}_{\tau}$ could be carefully studied at the Belle-II, SCTF or STCF, CEPC, FCC-ee, LHCb experiments. (3) For the $B_{u}^{*}$ mesons below the $B \pi$ thresholds and the $B_{c}^{*}$ mesons below both $B D$ and $B_{c} \pi$ thresholds, they decay predominantly through the magnetic dipole transitions. The branching ratios of the PLDCV $B_{c}^{*}$ decays favored by the CKM element $\left|V_{c b}\right|$ could reach up to $\mathcal{O}\left(10^{-6}\right)$. The PLDCV decays $B_{u, c}^{*} \rightarrow e^{-} \bar{v}_{e}, \mu^{-} \bar{v}_{\mu}, \tau^{-} \bar{v}_{\tau}$ might be searched for at the CEPC, FCC-ee, LHCb experiments. Our rough estimations and findings are summed in Table 7. We wish that our investigation could provoke physicists' researching interest in PLDCV and offer a ready reference for the future experimental analysis.

Acknowledgements The work is supported by the National Natural Science Foundation of China (Grant Nos. 11705047, 11981240403, U1632109, 11547014), the Chinese Academy of Sciences Large-Scale Scientific Facility Program (1G2017IHEPKFYJ01) and the Program for Innovative Research Team in University of Henan Province (19IRTSTHN018). We thank Prof. Haibo Li (IHEP@CAS), Prof. Shuangshi Fang (IHEP@CAS), Prof. Frank Porter (Caltech), Prof. Antimo Palano (INFN), Prof. Chengping Shen (Fudan University), Dr. Xiao Han (Fudan University), Prof. Xiaolin Kang (China University of Geosciences), Ms. Qingping Ji (Henan Normal University), Ms. Huijing Li (Henan Normal University) for their kindly help and valuable discussion.

Data Availability Statement This manuscript has no associated data or the data will not be deposited. [Authors' comment: All the data are completely inlcuded, properly cited and correctly deposited in our article, and can be officially published. We agree all the data to be deposited by the journal.]

Open Access This article is licensed under a Creative Commons Attribution 4.0 International License, which permits use, sharing, adaptation, distribution and reproduction in any medium or format, as long as you give appropriate credit to the original author(s) and the source, provide a link to the Creative Commons licence, and indicate if changes were made. The images or other third party material in this article are included in the article's Creative Commons licence, unless indicated otherwise in a credit line to the material. If material is not included in the article's Creative Commons licence and your intended use is not permitted by statutory regulation or exceeds the permitted use, you will need to obtain permission directly from the copy- right holder. To view a copy of this licence, visit http://creativecomm ons.org/licenses/by/4.0/.

Funded by $\mathrm{SCOAP}^{3}$.

\section{References}

1. P. Zyla et al. (Particle Data Group), Prog. Theor. Exp. Phys. 2020, 0801 (2020). https://doi.org/10.1093/ptep/ptaa104

2. M. Gell-Mann, Phys. Lett. 8, 214 (1964). https://doi.org/10.1016/ S0031-9163(64)92001-3

3. G. Zweig, CERN-TH-401, 402, 412 (1964)

4. G. Buchalla, A. Buras, M. Lautenbacher, Rev. Mod. Phys. 68, 1125 (1996). https://doi.org/10.1103/RevModPhys.68.1125

5. N. Cabibbo, Phys. Rev. Lett. 10, 531 (1963). https://doi.org/10. 1103/PhysRevLett.10.531

6. M. Kobayashi, T. Maskawa, Prog. Theor. Phys. 49, 652 (1973). https://doi.org/10.1143/PTP.49.652

7. P. Ball, V. Braun, A. Lenz, JHEP 05, 004 (2006). https://doi.org/ 10.1088/1126-6708/2006/05/004

8. P. Ball, J. Jones, JHEP 03, 069 (2007). https://doi.org/10.1088/ 1126-6708/2007/03/069

9. J. Hardy, I. Towner, PoS CKM2016, 028 (2016). https://doi.org/ $10.22323 / 1.291 .0028$

10. M. Tanabashi et al. (Particle Data Group), Phys. Rev. D 98, 030001 (2018). https://doi.org/10.1103/PhysRevD.98.030001

11. J. Forshaw, R. Sandapen, G. Shaw, Phys. Rev. D 69, 094013 (2004). https://doi.org/10.1103/PhysRevD.69.094013

12. M. McDermott, R. Sandapen, G. Shaw, Eur. Phys. J. C 22, 655 (2002). https://doi.org/10.1007/s100520100846

13. K. Golec-Biernat, M. Wusthoff, Phys. Rev. D 60, 114023 (1999). https://doi.org/10.1103/PhysRevD.60.114023

14. M. Ahmady, R. Sandapen, N. Sharma, Phys. Rev. D 94, 074018 (2016). https://doi.org/10.1103/PhysRevD.94.074018

15. A. Bakulev, S. Mikhailov, Phys. Lett. B 436, 351 (1998). https:// doi.org/10.1016/S0370-2693(98)00868-5

16. P. Ball, V. Braun, Phys. Rev. D 54, 2182 (1996). https://doi.org/ 10.1103/PhysRevD.54.2182

17. V. Chernyak, A. Zhitnitsky, Phys. Rep. 112, 173 (1984). https:// doi.org/10.1016/0370-1573(84)90126-1

18. P. Ball, V. Braun, Phys. Rev. D 58, 094016 (1998). https://doi.org/ 10.1103/PhysRevD.58.094016

19. P. Ball, R. Zwicky, JHEP 04, 046 (2006). https://doi.org/10.1088/ 1126-6708/2006/04/046

20. J. He, B. Juliá-Díaz, Y. Dong, Eur. Phys. J. A 24, 411 (2005). https://doi.org/10.1140/epja/i2005-10013-7

21. J. He, B. Juliá-Díaz, Y. Dong, Phys. Lett. B 602, 212 (2004). https://doi.org/10.1016/j.physletb.2004.10.004

22. D. Ebert, R. Faustov, V. Galkin, Phys. Lett. B 635, 93 (2006). https://doi.org/10.1016/j.physletb.2006.02.042 
23. H. Choi, C. Ji, Phys. Rev. D 75, 034019 (2007). https://doi.org/ 10.1103/PhysRevD.75.034019

24. H. Choi, C. Ji, Z. Li, H. Ryu, Phys. Rev. C 92, 055203 (2015). https://doi.org/10.1103/PhysRevC.92.055203

25. Q. Chang, X. Li, X. Li, F. Su, Chin. Phys. C 42, 073102 (2018). https://doi.org/10.1088/1674-1137/42/7/073102

26. R. Verma, J. Phys. G 39, 025005 (2012). https://doi.org/10.1088/ 0954-3899/39/2/025005

27. Q. Chang, X. Li, X. Li et al., Phys. Rev. D 98, 114018 (2018). https://doi.org/10.1103/PhysRevD.98.114018

28. N. Dhiman, H. Dahiya, C. Ji et al., Phys. Rev. D 100, 014026 (2019). https://doi.org/10.1103/PhysRevD.100.014026

29. A. Khan et al. (CP-PACS Collaboration), Phys. Rev. D 65, 054505 (2002). https://doi.org/10.1103/PhysRevD.65.054505

30. A. Khan et al. (CP-PACS Collaboration), Phys. Rev. D 67(E), 059901 (2003). https://doi.org/10.1103/PhysRevD.67.059901

31. K. Hashimoto, T. Izubuchi, Prog. Theor. Phys. 119, 599 (2008). https://doi.org/10.1143/PTP.119.599

32. K. Jansen, C. McNeile, C. Michael et al., Phys. Rev. D 80, 054510 (2009). https://doi.org/10.1103/PhysRevD.80.054510

33. V. Braun, P. Bruns, S. Collins et al., JHEP 04, 082 (2017). https:// doi.org/10.1007/JHEP04(2017)082

34. W. Sun et al. ( $\chi$ QCD Collaboration), Chin. Phys. C 42, 063102 (2018). https://doi.org/10.1088/1674-1137/42/6/063102

35. P. Maris, P. Tandy, Phys. Rev. C 60, 055214 (1999). https://doi. org/10.1103/PhysRevC.60.055214

36. Y. Simonov, Phys. Atom. Nucl. 79, 444 (2016). https://doi.org/ $10.1134 /$ S1063778816030169

37. Y. Grossman, M. König, M. Neubert, JHEP 04, 101 (2015). https://doi.org/10.1007/JHEP04(2015)101

38. W. Marciano, A. Sirlin, Phys. Rev. Lett. 61, 1815 (1988). https:// doi.org/10.1103/PhysRevLett.61.1815

39. E. Braaten, C. Li, Phys. Rev. D 42, 3888 (1990). https://doi.org/ 10.1103/PhysRevD.42.3888

40. B. Aubert et al. (BABAR Collaboration), Phys. Rev. D 78, 071103 (2008). https://doi.org/10.1103/PhysRevD.78.071103

41. C. Adamuščín, G. Gakh, E. Tomasi-Gustafsson, Phys. Rev. C 75, 065202 (2007). https://doi.org/10.1103/PhysRevC.75.065202

42. J. Melo, C. Ji, T. Frederico, Phys. Lett. B 763, 87 (2016). https:// doi.org/10.1016/j.physletb.2016.10.018

43. E. Kou et al., Prog. Theor. Exp. Phys. 123 C01 (2019). https://doi. org/10.1093/ptep/ptz106

44. E. Kou et al., Prog. Theor. Exp. Phys. 029201(E) (2020). https:// doi.org/10.1093/ptep/ptaa008

45. T. Uglov, EPJ Web Conf. 212, 01010 (2019). https://doi.org/10. 1051/epjconf/201921201010

46. C. Yuan, M. Karliner, Phys. Rev. Lett. 127, 0120031 (2021). https://doi.org/10.1103/PhysRevLett.127.012003

47. X. Shi, X. Zhou, X. Qin, H. Peng, JINST 16, P03029 (2021). https://doi.org/10.1088/1748-0221/16/03/P03029

48. A. Barnyakov et al., Nucl. Instrum. Methods Phys. Res. A 958, 162352 (2020). https://doi.org/10.1016/j.nima.2019.162352

49. H. Haber, J. Perrier, Phys. Rev. D 32, 2961 (1985). https://doi. org/10.1103/PhysRevD.32.2961

50. A. Bevan et al., Eur. Phys. J. C 74, 3026 (2014). https://doi.org/ 10.1140/epjc/s10052-014-3026-9

51. X. Kang, PoS EPS-HEP2019, 511 (2020). https://doi.org/10. 22323/1.364.0511

52. R. Aaij et al. (LHCb Collaboration), JHEP 10, 172 (2015). https:// doi.org/10.1007/JHEP10(2015)172

53. R. Aaij et al. (LHCb Collaboration), JHEP 05(E), 063 (2017). https://doi.org/10.1007/JHEP05(2017)063

54. I. Bediaga et al. (LHCb Collaboration), arXiv:1808.08865

55. R. Aaij et al. (LHCb Collaboration), JHEP 03, 159 (2016). https:// doi.org/10.1007/JHEP03(2015)159
56. R. Aaij et al. (LHCb Collaboration), JHEP 09(E), 013 (2016). https://doi.org/10.1007/JHEP09(2016)013

57. R. Aaij et al. (LHCb Collaboration), JHEP 05(E), 074 (2017). https://doi.org/10.1007/JHEP05(2017)074

58. A. Abada et al., Eur. Phys. J. Spec. Top. 228, 1109 (2019). https:// doi.org/10.1140/epjst/e2019-900088-6

59. P. Dimopoulos, F. Mescia, A. Vladikas, Phys. Rev. D 84, 014505 (2011). https://doi.org/10.1103/PhysRevD.84.014505

60. P. Vilain et al. (CHARM-II Collaboration), Eur. Phys. J. C 11, 19 (1999). https://doi.org/10.1007/s100529900141

61. C. Albertus, E. Hernández, J. Nieves et al., Phys. Rev. D 71, 113006 (2005). https://doi.org/10.1103/PhysRevD.71.113006

62. B. Yazarloo, H. Mehraban, EPL. 115, 21002 (2016). https://doi. org/10.1209/0295-5075/115/21002

63. B. Yazarloo, H. Mehraban, Eur. Phys. J. Plus 132, 80 (2017). https://doi.org/10.1140/epjp/i2017-11335-x

64. M. Abu-Shady, E. Khokha, Adv. High Energy Phys. 7032041 (2018). https://doi.org/10.1155/2018/7032041

65. D. Ebert, R. Faustov, V. Galkin, Mod. Phys. Lett. A 17, 803 (2002). https://doi.org/10.1142/S0217732302007077

66. D. Ebert, R. Faustov, V. Galkin, Phys. Rev. D 67, 014027 (2003). https://doi.org/10.1103/PhysRevD.67.014027

67. D. Hwang, G. Kim, Phys. Rev. D 55, 6944 (1997). https://doi.org/ 10.1103/PhysRevD.55.6944

68. H. Choi, Phys. Rev. D 75, 073016 (2007). https://doi.org/10.1103/ PhysRevD.75.073016

69. C. Hwang, Phys. Rev. D 81, 114024 (2010). https://doi.org/10. 1103/PhysRevD.81.114024

70. C. Geng, C. Lih, C. Xia, Eur. Phys. J. C 76, 313 (2016). https:// doi.org/10.1140/epjc/s10052-016-4172-z

71. N. Dhiman, H. Dahiya, Eur. Phys. J. Plus 133, 134 (2017). https:// doi.org/10.1140/epjp/i2018-11972-5

72. A. Martin, Phys. Lett. B 93, 338 (1980). https://doi.org/10.1016/ 0370-2693(80)90527-4

73. K. Hagiwara, A. Martin, A. Peacock, Z. Phys. C 33, 135 (1986). https://doi.org/10.1007/BF01410461

74. C. Quigg, J. Rosner, Phys. Lett. B 71, 153 (1977). https://doi.org/ 10.1016/0370-2693(77)90765-1

75. D. Becirevic, Ph. Boucaud, J. Leroy et al., Phys. Rev. D 60, 074501 (1999). https://doi.org/10.1103/PhysRevD.60.074501

76. K. Bowler et al. (UKQCD collaboration), Nucl. Phys. B 619, 507 (2001). https://doi.org/10.1016/S0550-3213(01)00511-9

77. D. Bečirević, V. Lubicz, F. Sanfilippo et al., JHEP 02, 042 (2012). https://doi.org/10.1007/JHEP02(2012)042

78. V. Lubicz, A. Melis, S. Simula, (ETM Collaboration), Phys. Rev. D 96, 034524 (2017). https://doi.org/10.1103/PhysRevD.96. 034524

79. Y. Chen ( $\chi$ QCD Collaboration), Chin. Phys. C 45, 023109 (2021). https://doi.org/10.1088/1674-1137/abcd8f

80. P. Gelhausen, A. Khodjamirian, A. Pivovarov et al., Phys. Rev. D 88, 014015 (2013). https://doi.org/10.1103/PhysRevD.88. 014015

81. P. Gelhausen, A. Khodjamirian, A. Pivovarov et al., Phys. Rev. D 89(E), 099901 (2013). https://doi.org/10.1103/PhysRevD.89. 099901

82. P. Gelhausen, A. Khodjamirian, A. Pivovarov et al., Phys. Rev. D 91(E), 099901 (2015). https://doi.org/10.1103/PhysRevD.91. 099901

83. Z. Wang, Eur. Phys. J. C 75, 427 (2015). https://doi.org/10.1140/ epjc/s10052-015-3653-9

84. W. Lucha, D. Melikhov, S. Simula, Phys. Lett. B 735, 12 (2014). https://doi.org/10.1016/j.physletb.2014.06.007

85. S. Narison, Int. J. Mod. Phys. A 30, 1550116 (2015). https://doi. org/10.1142/S0217751X1550116X

86. C. Albertus, E. Hernández, J. Nieves et al., Phys. Rev. D 75, 116001 (2007). https://doi.org/10.1103/PhysRevD.75.116001 
87. G. Wang, Phys. Lett. B 633, 492 (2006). https://doi.org/10.1103/ PhysRevD.81.114024

88. A. El-Hady, A. Datta, J. Vary, Phys. Rev. D 58, 014007 (1998). https://doi.org/10.1103/PhysRevD.58.014007

89. B. Zhou, J. Sun, Y. Zhang, Commun. Theor. Phys. 67, 655 (2017). https://doi.org/10.1088/0253-6102/67/6/655

90. V. Zhukova et al. (Belle Collaboration), Phys. Rev. D 97, 012002 (2018). https://doi.org/10.1103/PhysRevD.97.012002

91. B. Aubert et al. (BaBar Collaboration), Phys. Rev. D 79, 071103 (2009). https://doi.org/10.1103/PhysRevD.79.092001

92. D. Cronin-Hennessy et al. (CLEO Collaboration), Phys. Rev. D 80, 072001 (2009). https://doi.org/10.1103/PhysRevD.80. 072001

93. X. Dong, L. Wang, C. Yuan, Chin. Phys. C 42, 043002 (2018). https://doi.org/10.1088/1674-1137/42/4/043002

94. J. Costa et al., IHEP-CEPC-DR-2018-02. arXiv:1811.10545

95. A. Abada et al., Eur. Phys. J. C 79, 474 (2019). https://doi.org/10. 1140/epjc/s10052-019-6904-3

96. B. Abelev et al. (ALICE Collaboration), JHEP 07, 191 (2012). https://doi.org/10.1007/JHEP07(2012)191

97. G. Aad et al. (ATLAS Collaboration), Nucl. Phys. B 907, 117 (2016). https://doi.org/10.1016/j.nuclphysb.2016.04.032

98. L. Gladilin, Eur. Phys. J. C 75, 19 (2015). https://doi.org/10.1140/ epjc/s10052-014-3250-3

99. S. Acharya et al. (ALICE Collaboration), arXiv:2108.02523

100. S. Okubo, Phys. Lett. 5, 165 (1963). https://doi.org/10.1016/ S0375-9601(63)92548-9

101. J. Iizuka, Prog. Theor. Phys. Suppl. 37-38, 21 (1966). https://doi. org/10.1143/PTPS.37.21

102. M. Ablikim et al. (BESIII Collaboration), Phys. Rev. D 104, 052009 (2021). https://doi.org/10.1103/PhysRevD.104.052009

103. N. Soni, J. Pandya, Phys. Rev. D 96, 016017 (2017). https://doi. org/10.1103/PhysRevD.96.016017

104. N. Soni, J. Pandya, Phys. Rev. D 99(E), 059901 (2019). https:// doi.org/10.1103/PhysRevD.99.059901

105. J. Gronberg et al. (CLEO Collaboration), Phys. Rev. Lett. 75, 3232 (1995). https://doi.org/10.1103/PhysRevLett.75.3232

106. G. Donald, C. Davies, J. Koponen, G. Lepage, Phys. Rev. Lett. 112, 212002 (2014). https://doi.org/10.1103/PhysRevLett.112. 212002

107. R. Hackman, N. Deshpande, D. Dicus et al., Phys. Rev. D 18, 2537 (1978). https://doi.org/10.1103/PhysRevD.18.2537

108. E. Eichten, K. Gottfried, T. Kinoshita et al., Phys. Rev. D 21, 203 (1980). https://doi.org/10.1103/PhysRevD.21.203

109. W. Wilcox, O. Maxwell, K. Milton, Phys. Rev. D 31, 1081 (1985). https://doi.org/10.1103/PhysRevD.31.1081

110. G. Miller, P. Singer, Phys. Rev. D 37, 2564 (1988). https://doi. org/10.1103/PhysRevD.37.2564

111. A. Kamal, P. Xu, Phys. Lett. B 284, 421 (1992). https://doi.org/ 10.1016/0370-2693(92)90455-D

112. T. Aliev, E. Iltan, N. Pak, Phys. Lett. B 334, 169 (1994). https:// doi.org/10.1016/0370-2693(94)90606-8

113. P. O'Donnell, P. Xu, Phys. Lett. B 336, 113 (1994). https://doi. org/10.1016/0370-2693(94)00975-9

114. G. Burdman, E. Golowich, J. Hewett et al., Phys. Rev. D 52, 6383 (1995). https://doi.org/10.1103/PhysRevD.52.6383

115. F. Close, E. Swanson, Phys. Rev. D 72, 094004 (2005). https:// doi.org/10.1103/PhysRevD.72.094004

116. G. Yu, Z. Li, Z. Wang, Eur. Phys. J. C 75, 243 (2015). https://doi. org/10.1140/epjc/s10052-015-3460-3

117. N. Li, Y. Wu, Int. J. Mod. Phys. A 31, 1650109 (2016). https:// doi.org/10.1142/S0217751X16501098

118. B. Yang, B. Wang, L. Meng et al., Phys. Rev. D 101, 054019 (2020). https://doi.org/10.1103/PhysRevD.101.054019

119. H. Cheng, C. Cheung, G. Lin et al., Phys. Rev. D 47, 1030 (1993). https://doi.org/10.1103/PhysRevD.47.1030
120. P. Colangelo, F. Fazio, G. Nardulli, Phys. Lett. B 316, 555 (1993). https://doi.org/10.1016/0370-2693(93)91043-M

121. P. Colangelo, F. Fazio, G. Nardulli, Phys. Lett. B 334, 175 (1994). https://doi.org/10.1016/0370-2693(94)90607-6

122. N. Barik, P. Dash, Phys. Rev. D 49, 299 (1994). https://doi.org/ 10.1103/PhysRevD.49.299

123. N. Barik, P. Dash, Phys. Rev. D 53(E), 4110 (1996). https://doi. org/10.1103/PhysRevD.53.4110

124. M. Ivanov, Y. Valit, Z. Phys. C 67(633) (1995). https://doi.org/10. 1007/BF01553990

125. S. Zhu, Z. Yang, Mod. Phys. Lett. A 12, 3027 (1997). https://doi org/10.1142/S0217732397003150

126. S. Jena, S. Panda, T. Tripathy, Nucl. Phys. A 658, 249 (1999). https://doi.org/10.1016/S0375-9474(99)00332-2

127. Y. Dong, A. Faessler, K. Shimizu, Nucl. Phys. A 671, 380 (2000) https://doi.org/10.1016/S0375-9474(99)00822-2

128. S. Jena, P. Panda, K. Sahu, J. Phys. G 27, 1519 (2001). https:// doi.org/10.1088/0954-3899/27/7/311

129. J. Goity, W. Roberts, Phys. Rev. D 64, 094007 (2001). https://doi. org/10.1103/PhysRevD.64.094007

130. R. Bonnaz, B. Silvestre-Brac, C. Gignoux, Eur. Phys. J. A 13, 363 (2002). https://doi.org/10.1007/s10050-002-8765-6

131. D. Ebert, R. Faustov, V. Galkin, Phys. Lett. B 537, 241 (2002) https://doi.org/10.1016/S0370-2693(02)01939-1

132. W. Bardeen, E. Eichten, C. Hill, Phys. Rev. D 68, 054024 (2003). https://doi.org/10.1103/PhysRevD.68.054024

133. S. Jena, M. Muni, H. Pattnaik et al., Int. J. Mod. Phys. A 25, 2063 (2010). https://doi.org/10.1142/S0217751X10048214

134. V. Šimonis, Eur. Phys. J. A 52, 90 (2016). https://doi.org/10.1140/ epja/i2016-16090-5

135. M. Priyadarsini, P. Dash, S. Kar et al., Phys. Rev. D 94, 113011 (2016). https://doi.org/10.1103/PhysRevD.94.113011

136. B. Wang, B. Yang, L. Meng et al., Phys. Rev. D 100, 016019 (2019). https://doi.org/10.1103/PhysRevD.100.016019

137. H. Li, C. Lü, C. Wang et al., JHEP 04, 023 (2020). https://doi. org/10.1007/JHEP04(2020)023

138. B. Pullin, R. Zwicky, JHEP 09, 023 (2021)

139. R. Fayyazuddin, A modern introduction to particle physics (2011). https://doi.org/10.1142/8064

140. G. Pakhlova et al. (Belle Collaboration), Phys. Rev. D 83, 011101 (2011). https://doi.org/10.1103/PhysRevD.83.011101

141. P. Sanchez et al. (BaBar Collaboration), Phys. Rev. D 82, 052004 (2010). https://doi.org/10.1103/PhysRevD.82.052004

142. M. Lisovyi, A. Verbytskyi, O. Zenaiev, Eur. Phys. J. C 76, 397 (2016). https://doi.org/10.1140/epjc/s10052-016-4246-y

143. B. Yazarloo, H. Mehraban, EPL 116, 31004 (2016). https://doi. org/10.1209/0295-5075/116/31004

144. B. Colquhoun et al. (HPQCD Collaboration), Phys. Rev. D 91, 114509 (2015). https://doi.org/10.1103/PhysRevD.91.114509

145. W. Lucha, D. Melikhov, S. Simula, Phys. Rev. D 91, 116009 (2015). https://doi.org/10.1103/PhysRevD.91.116009

146. J. Abdallah et al. (DELPHI Collaboration), Phys. Lett. B 576, 29 (2003). https://doi.org/10.1016/j.physletb.2003.09.070

147. R. Aaij et al. (LHCb Collaboration), JHEP 07, 123 (2020). https:// doi.org/10.1007/JHEP07(2020)123

148. R. Aaij et al. (LHCb Collaboration), Phys. Rev. Lett. 122, 232001 (2019). https://doi.org/10.1103/PhysRevLett.122.232001

149. A. Sirunyan et al. (CMS Collaboration), Phys. Rev. Lett. 122, 132001 (2019). https://doi.org/10.1103/PhysRevLett.122. 132001

150. S. Nussinov, Z. Phys. C 3, 165 (1979). https://doi.org/10.1007/ BF01443703

151. D. Stanley, D. Robson, Phys. Rev. D 21, 3180 (1980). https://doi. org/10.1103/PhysRevD.21.3180

152. E. Eichten, F. Feinberg, Phys. Rev. D 23, 2724 (1981). https://doi. org/10.1103/PhysRevD.23.2724 
153. W. Buchmüller, S. Tye, Phys. Rev. D 24, 132 (1981). https://doi. org/10.1103/PhysRevD.24.132

154. A. Kaidalov, Z. Phys. C 12(63) (1982). https://doi.org/10.1007/ BF01475732

155. S. Godfrey, N. Isgur, Phys. Rev. D 32, 189 (1985). https://doi.org/ 10.1103/PhysRevD.32.189

156. S. Gershtein, A. Likhoded, S. Slabospitsky, Int. J. Mod. Phys. A 6, 2309 (1991). https://doi.org/10.1142/S0217751X91001131

157. W. Kwong, J. Rosner, Phys. Rev. D 44, 212 (1991). https://doi. org/10.1103/PhysRevD.44.212

158. Y. Chen, Y. Kuang, Phys. Rev. D 46, 1165 (1992). https://doi.org/ 10.1103/PhysRevD.46.1165

159. Y. Chen, Y. Kuang, Phys. Rev. D 47(E), 350 (1993). https://doi. org/10.1103/PhysRevD.47.350

160. E. Eichten, C. Quigg, Phys. Rev. D 49, 5845 (1994). https://doi. org/10.1103/PhysRevD.49.5845

161. E. Bagan, H. Dosch, P. Gosdzinsky et al., Z. Phys. C 64, 57 (1994). https://doi.org/10.1007/BF01557235

162. S. Gershtein, V. Kiselev, A. Likhoded et al., Phys. Usp. 38, 1 (1995). https://doi.org/10.1070/PU1995v038n01ABEH000063

163. R. Roncaglia, A. Dzierba, D. Lichtenberg et al., Phys. Rev. D 51, 1248 (1995). https://doi.org/10.1103/PhysRevD.51.1248

164. S. Gershtein, V. Kiselev, A. Likhoded et al., Phys. Rev. D 51, 3613 (1995). https://doi.org/10.1103/PhysRevD.51.3613

165. J. Zeng, J. Orden, W. Roberts, Phys. Rev. D 52, 5229 (1995). https://doi.org/10.1103/PhysRevD.52.5229

166. S. Gupta, J. Johnson, Phys. Rev. D 53, 312 (1996). https://doi.org/ 10.1103/PhysRevD.53.312

167. C. Davies, K. Hornbostel, G. Lepage et al., Phys. Lett. B 382, 131 (1996). https://doi.org/10.1016/0370-2693(96)00650-8

168. L. Motyka, K. Zalewski, Eur. Phys. J. C 4, 107 (1998). https:// doi.org/10.1007/s100520050190

169. A. El-Hady, M. Lodhi, J. Vary, Phys. Rev. D 59, 094001 (1999). https://doi.org/10.1103/PhysRevD.59.094001

170. L. Fulcher, Phys. Rev. D 60, 074006 (1999). https://doi.org/10. 1103/PhysRevD.60.074006

171. M. Baldicchi, G. Prosperi, Phys. Rev. D 62, 114024 (2000). https://doi.org/10.1103/PhysRevD.62.114024

172. S. Godfrey, Phys. Rev. D 70, 054017 (2004). https://doi.org/10. 1103/PhysRevD.70.054017

173. S. Ikhdair, R. Sever, Int. J. Mod. Phys. A 19, 1771 (2004). https:// doi.org/10.1142/S0217751X0401780X

174. D. Li, B. Ma, Y. Li et al., Eur. Phys. J. C 37, 323 (2004). https:// doi.org/10.1140/epjc/s2004-02002-5

175. A. El-Hady, J. Spence, J. Vary, Phys. Rev. D 71, 034006 (2005). https://doi.org/10.1103/PhysRevD.71.034006

176. A. Rai, P. Vinodkumar, Pramana 66, 953 (2006). https://doi.org/ 10.1007/BF02704795

177. T. Chiu et al. (TWQCD Collaboration), Phys. Lett. B 651, 171 (2007). https://doi.org/10.1016/j.physletb.2007.06.017

178. A. Rai, B. Patel, P. Vinodkumar, Phys. Rev. C 78, 055202 (2008). https://doi.org/10.1103/PhysRevC.78.055202

179. B. Patel, P. Vinodkumar, J. Phys. G 36, 035003 (2009). https:// doi.org/10.1088/0954-3899/36/3/035003

180. A. Parmar, B. Patel, P. Vinodkumar, Nucl. Phys. B 848, 299 (2010). https://doi.org/10.1016/j.nuclphysa.2010.08.016

181. K. Wei, X. Guo, Phys. Rev. D 81, 076005 (2010). https://doi.org/ 10.1103/PhysRevD.81.076005

182. E. Gregory et al. (HPQCD Collaboration), Phys. Rev. Lett. 104, 022001 (2010). https://doi.org/10.1103/PhysRevLett.104. 022001

183. A. Badalian, B. Bakker, I. Danilkin, Phys. Rev. D 81, 071502 (2010). https://doi.org/10.1103/PhysRevD.81.071502

184. A. Badalian, B. Bakker, I. Danilkin, Phys. Rev. D 81(E), 099902 (2010). https://doi.org/10.1103/PhysRevD.81.099902
185. D. Ebert, R. Faustov, V. Galkin, Eur. Phys. J. C 71, 1825 (2011). https://doi.org/10.1140/epjc/s10052-011-1825-9

186. A. Badalian, B. Bakker, I. Danilkin, Phys. Atom. Nucl. 74, 631 (2011). https://doi.org/10.1134/S1063778811040028

187. R. Dowdall, C. Davies, T. Hammant et al., Phys. Rev. D 86, 094510 (2012). https://doi.org/10.1103/PhysRevD.86.094510

188. Z. Wang, Eur. Phys. J. A 49, 131 (2013). https://doi.org/10.1140/ epja/i2013-13131-7

189. N. Devlani, V. Kher, A. Rai, Eur. Phys. J. A 50, 154 (2014). https:// doi.org/10.1140/epja/i2014-14154-2

190. A. Monteiro, M. Bhat, K. Kumar, Int. J. Mod. Phys. A 32, 1750021 (2017). https://doi.org/10.1142/S0217751X1750021X

191. A. Monteiro, M. Bhat, K. Kumar, Phys. Rev. D 95, 054016 (2017) https://doi.org/10.1103/PhysRevD.95.054016

192. N. Mathur, M. Padmanath, S. Mondal, Phys. Rev. Lett 121, 202002 (2018). https://doi.org/10.1103/PhysRevLett.121. 202002

193. N. Soni, B. Joshi, R. Shah et al., Eur. Phys. J. C 78, 592 (2018). https://doi.org/10.1140/epjc/s10052-018-6068-6

194. E. Eichten, C. Quigg, Phys. Rev. D 99, 054025 (2019). https:// doi.org/10.1103/PhysRevD.99.054025

195. Q. Li, M. Liu, L. Lu et al., Phys. Rev. D 99, 096020 (2019). https:// doi.org/10.1103/PhysRevD.99.096020

196. I. Asghar, F. Akram, B. Masud et al., Phys. Rev. D 100, 096002 (2019). https://doi.org/10.1103/PhysRevD.100.096002

197. L. Gutiérrez-Guerrero, A. Bashir, A. Bedolla et al., Phys. Rev. D 100, 114032 (2019). https://doi.org/10.1103/PhysRevD.100. 114032

198. N. Akbar, F. Akram, B. Masud et al., Eur. Phys. J. A 55, 82 (2019). https://doi.org/10.1140/epja/i2019-12735-1

199. T. Aliev, T. Barakat, S. Bilmis, Nucl. Phys. B 947, 114726 (2019). https://doi.org/10.1016/j.nuclphysb.2019.114726

200. P. Ortega, J. Segovia, D. Entem et al., Eur. Phys. J. C 80, 223 (2020). https://doi.org/10.1140/epjc/s10052-020-7764-6

201. S. Narison, Phys. Lett. B 807, 135522 (2020). https://doi.org/10. 1016/j.physletb.2020.135522

202. M. Chen, L. Chang, Y. Liu, Phys. Rev. D 101, 056002 (2020). https://doi.org/10.1103/PhysRevD.101.056002

203. L. Abreu, F. Júnior, A. Favero, Phys. Rev. D 102, 034002 (2020). https://doi.org/10.1103/PhysRevD.102.034002

204. N. Akbar, Phys. Atom. Nucl. 83, 634 (2020). https://doi.org/10. 1134/S1063778820040031

205. R. Ding, B. Wan, Z. Chen et al., Phys. Lett. B 816, 136277 (2021). https://doi.org/10.1016/j.physletb.2021.136277

206. J. Ahmed, R. Manzoor, L. Chang et al., Few-Body Syst. 62, 39 (2021). https://doi.org/10.1007/s00601-021-01624-1

207. P. Yin, Z. Cui, C. Roberts et al., Eur. Phys. J. C 81, 327 (2021). https://doi.org/10.1140/epjc/s10052-021-09097-6

208. H. Choi, C. Ji, Phys. Rev. D 80, 054016 (2009). https://doi.org/ 10.1103/PhysRevD.80.054016

209. S. Tang, Y. Li, P. Maris et al., Phys. Rev. D 98, 114038 (2018). https://doi.org/10.1103/PhysRevD.98.114038

210. D. Becirevic et al. (ETM Collaboration), PoS LATTICE2018, 273 (2019). https://doi.org/10.22323/1.334.0273

211. G. Boroun, S. Zarrin, S. Dadfar, Nucl. Phys. A 953, 21 (2016). https://doi.org/10.1016/j.nuclphysa.2016.03.032

212. D. Yang, W. zhang, Chin. Phys. C 43, 083101 (2019). https://doi org/10.1088/1674-1137/43/8/083101

213. X. Zheng, C. Chang, T. Feng et al., Phys. Rev. D 100, 034004 (2019). https://doi.org/10.1103/PhysRevD.100.034004

214. G. Chen, C. Chang, X. Wu, Phys. Rev. D 97, 114022 (2018). https://doi.org/10.1103/PhysRevD.97.114022 